\title{
HIGHER NON-ABELIAN COHOMOLOGY OF GROUPS
}

\author{
HVEDRI INASSARIDZE \\ A. Razmadze Matematical Institute, Georgian Academy of Sciences, Georgia \\ e-mail: hvedri@rmi.acnet.ge \\ URL: http://www.rmi.acnet.ge/ hvedri/
}

(Received 21 February, 2001; accepted 17 July 2001)

\begin{abstract}
The first non-abelian cohomology of groups introduced by Guin is extended to any dimensions and for a substantially wider class of coefficients called G-partially crossed P-modules. The first and the second non-abelian cohomologies of groups are described in terms of torsors and extensions of groups respectively. Higher non-abelian cohomology pointed sets are described in terms of cotriple right derived functors of the group of derivations with respect to the first contravariant variable. For any short exact coefficient sequence a long exact cohomology sequence is obtained extending the well-known exact cohomology sequences and higher cohomology of groups with coefficients in any G-group is introduced.
\end{abstract}

2000 Mathematics Subject Classification. 18G50, 18G55.

0. Introduction. Our approach to non-abelian cohomology of groups follows Guin's first non-abelian cohomology $[\mathbf{5 , 6}]$ which differs from the classical first nonabelian cohomology pointed set [10] and from the setting of various papers on nonabelian cohomology $[\mathbf{4 , 2}, \mathbf{3}]$ extending the classical exact non-abelian cohomology sequence from lower dimensions [10] to higher dimensions.

Guin defined his first non-abelian cohomology group when the coefficient group is a crossed $G$-module and obtained a six term exact cohomology sequence for any short exact sequence of crossed $G$-modules.

A non-abelian cohomology of groups will be defined in any dimension greater than 0, extending Guin's first non-abelian cohomology group and his exact cohomology sequence to a nine term exact cohomology sequence. A substantially wider class of coefficients will be used, consisting of partially crossed modules over a group $P$ on which a group $G$ acts on the left: these will be called $G$-partially crossed modules over $P$. We describe the first non-abelian cohomology in terms of torsors and the second non-abelian cohomology in terms of extensions of groups. Moreover a close relation between non-abelian cohomology of groups and nonabelian right derived functors of the group of derivations will be established and for some particular cases of coefficients a long exact cohomology sequence will be obtained.

All considered groups will be arbitrary (not necessarily commutative). An action of a group $G$ on a group $A$ means an action on the left of $G$ on $A$ by automorphisms and will be denoted by ${ }^{g} a, g \in G, a \in A$. We assume that $G$ acts on itself by conjugation. The center of a group $G$ will be denoted by $Z(G)$. If the groups $G$ and $R$ act on a group $A$ then the notation ${ }^{g r} a$ means ${ }^{g}\left({ }^{r} a\right), g \in G, r \in R, a \in A$. 
1. $G$-partially crossed $P$-modules and the group $\operatorname{Der}(G,(A, \mu))$ of derivations. A precrossed $P$-module $(A, \mu)$ consists of a group $P$ acting on a group $A$ and a homomorphism $\mu: A \longrightarrow P$ such that

$$
\mu\left({ }^{x} a\right)=x \mu(a) x^{-1}, \quad x \in P, a \in A .
$$

If in addition we have

$$
{ }^{\mu(a)} a^{\prime}=a a^{\prime} a^{-1}
$$

for $a, a^{\prime} \in A$, then $(A, \mu)$ is a crossed $P$-module.

Definition 1.1. A partially crossed module $\mu: A \longrightarrow P$ over $P$ is a precrossed module over $P$ satisfying the equality

$$
a a^{\prime} a^{-1}={ }^{\mu(a)} a^{\prime}
$$

for all $a^{\prime} \in A$ and for all $a \in A$ such that $\mu(a)$ is a commutator of $P$.

Note that the relation (1.1) is equivalent to the following relation:

$$
a^{y x} a^{\prime}={ }^{x y} a^{\prime} a,
$$

for all $a^{\prime} \in A$ and for all $a \in A$ such that $\mu(a)=x y x^{-1} y^{-1}$. To see that (1.1) implies (1.2) take $a^{\prime}={ }^{y x} b$ and that (1.2) implies (1.1) take $a^{\prime}=x^{x^{-1} y^{-1}} b$. Clearly any crossed module over $P$ is a partially $P$-crossed $P$-module.

Let $A$ be a metabelian (not abelian) group. Consider the precrossed module

$$
A \stackrel{\tau}{\longrightarrow} A /[A, A]=P,
$$

where $\tau$ is the canonical surjection and $P$ acts trivially on $A$. Then $A \stackrel{\tau}{\rightarrow} P$ is a partially crossed module over $P$ which is not a crossed $P$-module.

Any precrossed module $B \stackrel{\mu}{\rightarrow} P$ induces in a natural way a partially crossed module over $P$ as follows. Consider the Peiffer commutators $b b^{\prime} b^{-1} \mu(b) b^{-1}$ for all $b^{\prime} \in B$ subject to the relation: $\mu(b)$ is a commutator of $P$. Let $N$ be the normal subgroup of $B$ generated by these Peiffer commutators and take the quotient group $B / N$. One gets a precrossed module $B / N \stackrel{\mu^{\prime}}{\rightarrow} P, \mu^{\prime}$ being induced by $\mu$. It is easy to check that in fact it is a partially crossed $P$-module. Moreover any morphism from $B \stackrel{\mu}{\rightarrow} P$ to a partially crossed module $X \stackrel{v}{\rightarrow} C$ induces in a natural way a unique morphism

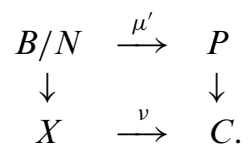

It is obvious that if $A \stackrel{\mu}{\rightarrow} P$ is a partially $P$-crossed module, then $\operatorname{Ker} \mu$ is contained in the center of $A$. 
Definition 1.2. Let $G, P$ and $A$ be groups. It will be said that $A \stackrel{\mu}{\rightarrow} P$ is a $G$-precrossed module over $P$ if

(1) $(A, \mu)$ is a precrossed $P$-module,

(2) $G$ acts on $P$ and $A$,

(3) $\mu: A \longrightarrow P$ is a homomorphism of $G$-groups,

(4) ${ }^{\left.{ }^{g} x\right)} a={ }^{g x g^{-1}} a$ for $g \in G, x \in P, a \in A$ (compatibility condition).

If in addition $(A, \mu)$ is a crossed $P$-module, then $(A, \mu)$ is called a $G$-crossed $P$-module. If conditions (1)-(4) hold it will be said that the group $G$ acts on the precrossed $P$-module $(A, \mu)$.

Definition 1.3. A $G$-precrossed $P$-module $(A, \mu)$ will be called a $G$-partially crossed $P$-module if in addition the following condition holds:

$$
a a^{\prime} a^{-1}={ }^{\mu(a)} a^{\prime} \quad \text { or equivalently } a^{y x} a^{\prime}={ }^{x y} a^{\prime} a,
$$

for all $a^{\prime} \in A$ and $a \in A$ such that $\mu(a)=x y x^{-1} y^{-1}$ for some $x, y \in P$.

It is clear that any precrossed (crossed) $G$-module is in a natural way a $G$-precrossed ( $G$-crossed) $G$-module, $G$ acting on itself by conjugation. A $G$-precrossed $P$ module was called in [8] a precrossed $G-P$-bimodule, causing confusion with the notion of crossed bimodule defined in [9, E.1.5.1] generalising the well-known notion of bimodule. If $f: G^{\prime} \longrightarrow G$ is a homomorphism of groups then any $G$-precrossed $P$-module is a $G^{\prime}$-precrossed $P$-module induced by $f, G^{\prime}$ acting on $A$ and $P$ via $f$.

Note that if $(A, \mu)$ is a $G$-precrossed $P$-module the equality

$$
{ }^{r x} a={ }^{x r} a
$$

holds for any $x \in G, a \in A, r \in H^{0}(G, P)$. In effect, one has

$$
{ }^{r x} a={ }^{x x^{-1} r x} a={ }^{x\left({ }^{-1} r\right)} a={ }^{x r} a .
$$

Definition 1.4. Let $(A, \mu)$ be a $G$-partially crossed $P$-module. Denote by $\operatorname{Der}(G,(A, \mu))$ the set of pairs $(\alpha, r)$, where $\alpha$ is a crossed homomorphism from $G$ to $A$, that is

$$
\alpha(x y)=\alpha(x){ }^{x} \alpha(y), \quad x, y \in G,
$$

and $r$ is an element of $P$ such that

$$
\mu \alpha(x)=r^{x} r^{-1}, \quad x \in G .
$$

This set will be called the set of derivations from $G$ to $(A, \mu)$.

For any $(\alpha, r) \in \operatorname{Der}(G,(A, \mu))$ one has

$$
\alpha(x){ }^{x r} a={ }^{r x} \alpha \alpha(x),
$$

for $a \in A, x \in G$. 
We introduce in $\operatorname{Der}(G,(A, \mu))$ a product by

$$
(\alpha, r)(\beta, s)=(\alpha * \beta, r s)
$$

where $(\alpha * \beta)(x)={ }^{r} \beta(x) \alpha(x), x \in G$.

Proposition 1.5. Under the aforementioned product, $\operatorname{Der}(G,(A, \mu))$ is a group which coincides with the group $\operatorname{Der}_{G}(G, A)$ of Guin if $(A, \mu)$ is a crossed G-module viewed as a G-crossed G-module.

Proof. Clearly this product is associative. First it will be shown that $(\alpha * \beta, r s)$ belongs to $\operatorname{Der}(G,(A, \mu))$. Put $\gamma=\alpha * \beta$. We have

$$
\begin{aligned}
\gamma(x y)={ }^{r} \beta(x y) \alpha(x y) & ={ }^{r}\left(\beta(x)^{x} \beta(y)\right) \alpha(x)^{x} \alpha(y) \\
& ={ }^{r} \beta(x)^{r x} \beta(y) \alpha(x)^{x} \alpha(y) .
\end{aligned}
$$

On the other hand

$$
\gamma(x)^{x} \gamma(y)^{\bullet}={ }^{r} \beta(x) \alpha(x)^{x}\left({ }^{r} \beta(y) \alpha(y)\right)={ }^{r} \beta(x) \alpha(x)^{x r} \beta(y)^{x} \alpha(y) .
$$

Using equality (1.1) one gets $\gamma(x y)=\gamma(x)^{x} \gamma(y)$ showing that $\gamma$ is a crossed homomorphism. Further, we have

$$
\begin{aligned}
\mu \gamma(x) & =\mu\left({ }^{r} \beta(x) \alpha(x)\right)={ }^{r} \mu \beta(x) \mu \alpha(x)={ }^{r}\left(s^{x} s^{-1}\right) r^{x} r^{-1}={ }^{r} s^{r}\left(s^{-1}\right) r \\
{ }^{x} r^{-1} & ={ }^{r} s r^{x} s^{-1}{ }^{-1}=r s^{x}(r s)^{-1} .
\end{aligned}
$$

Therefore $(\alpha * \beta, r s) \in \operatorname{Der}(G,(A, \mu))$. It is obvious that $\left(\alpha_{0}, 1\right)$ is the unit of $\operatorname{Der}(G,(A, \mu))$ with $\alpha_{0}(x)=1$ for all $x \in G$.

For $(\alpha, r) \in \operatorname{Der}(G,(A, \mu))$ take the pair $\left(\bar{\alpha}, r^{-1}\right)$ with $\bar{\alpha}(x)={ }^{r^{-1}} \alpha(x)^{-1}, x \in G$. It will be shown that $\left(\bar{\alpha}, r^{-1}\right) \in \operatorname{Der}(G,(A, \mu))$. For this the equality

$$
{ }^{r^{-1} x} a \cdot{ }^{r^{-1}} \alpha(x)^{-1}={ }^{r^{-1}} \alpha(x)^{-1} \cdot{ }^{x r^{-1}} a, x \in G, a \in A
$$

will be proved. In effect, since $\mu\left(r^{-1} \alpha(x)^{-1}\right)=r^{-1} \mu \alpha(x)^{-1} r=r^{-1 x} r$, one gets

$$
\mu\left(r^{-1} \alpha(x)^{-1}\right)\left({ }^{x-1} a\right)={ }^{r^{-1 x}} r\left(x r^{-1} a\right)={ }^{r^{-1} x r x^{-1} x r^{-1}} a={ }^{r^{-1} x} a \text {. }
$$

The required relation (1.3) follows now from the equality

$$
\mu\left(r^{-1} \alpha(x)^{-1}\right)\left({ }^{x r^{-1}} a\right)=r^{-1} \alpha(x)^{x r^{-1}} a^{r^{-1}} \alpha(x) .
$$

Therefore one has

$$
\begin{aligned}
\bar{\alpha}(x y) & ={ }^{r^{-1}} \alpha(x y)^{-1}={ }^{r^{-1}}\left({ }^{x} \alpha(y)^{-1} \alpha(x)^{-1}\right)={ }^{r^{-1} x} \alpha(y)^{-1 r^{-1}} \alpha(x)^{-1} \\
& ={ }^{r^{-1}} \alpha(x)^{-1 x r^{-1}} \alpha(y)^{-1}=\bar{\alpha}(x)^{x} \bar{\alpha}(y),
\end{aligned}
$$

that is $\bar{\alpha}$ is a crossed homomorphism. Moreover, 


$$
\mu \bar{\alpha}(x)=\mu\left({ }^{r^{-1}} \alpha(x)^{-1}\right)=r^{-1} \mu \alpha(x)^{-1} r=r^{-1}{ }^{x} r r^{-1} r=r^{-1 x} r .
$$

It follows that $\left(\bar{\alpha}, r^{-1}\right) \in \operatorname{Der}(G,(A, \mu))$. It is easily checked that

$$
(\alpha, r)\left(\bar{\alpha}, r^{-1}\right)=\left(\bar{\alpha}, r^{-1}\right)(\alpha, r)=\left(\alpha_{0}, 1\right) .
$$

We conclude that $\operatorname{Der}(G,(A, \mu))$ is a group which coincides with the group $\operatorname{Der}_{G}(G, A)$ of derivations defined by Guin [6] when $(A, \mu)$ is a crossed $G$-module.

A homomorphism $f:(A, \mu) \longrightarrow(B, \lambda)$ of $G$-partially crossed $P$-modules induces a homomorphism

$$
f^{*}: \operatorname{Der}(G,(A, \mu)) \longrightarrow \operatorname{Der}(G,(B, \lambda))
$$

given by $(\alpha, r) \mapsto(\alpha f, r)$.

There is an action of $G$ on $\operatorname{Der}(G,(A, \mu))$ defined by

$$
{ }^{g}(\alpha, r)=\left(\bar{\alpha},{ }^{g} r\right), \quad g \in G, r \in P,
$$

with $\bar{\alpha}(x)={ }^{g} \alpha\left({ }^{g^{-1}} x\right), x \in G$ (see $\left.[6,8]\right)$. Moreover if $P$ acts on $G$ such that

$$
{ }^{\left({ }^{g} g\right)} a={ }^{r g r^{-1}} a, \quad{ }^{\left({ }^{r} g\right)} r^{\prime}={ }^{r g r^{-1}} r^{\prime} \text { for } r, r^{\prime} \in R, g \in G, a \in A,
$$

then there is also an action of $P$ on $\operatorname{Der}(G,(A, \mu))$ given by ${ }^{r}(\alpha, s)=\left(\bar{\alpha},{ }^{r} s\right)$, $\bar{\alpha}(x)={ }^{r} \alpha\left({ }^{-1} x\right), r \in P, x \in G[8]$.

It is well-known [1] that the groups $G$ and $P$ acting on each other and on themselves by conjugation are said to be acting compatibly if

$$
{ }^{\left({ }^{g} r\right)} g^{\prime}={ }^{g r g^{-1}} g^{\prime}, \quad{ }^{\left({ }^{r} g\right)} r^{\prime}={ }^{r g} r^{-1} r^{\prime} \quad \text { for } g, g^{\prime} \in G, r, r^{\prime} \in R .
$$

Definition 1.6. It will be said that the groups $G$ and $P$ act on a group $A$ compatibly if

$$
{ }^{\left({ }^{g} r\right)} a={ }^{g r g^{-1}} a, \quad{ }^{\left({ }^{r} g\right)} a={ }^{r g r^{-1}} a \text { for } g \in G, r \in R, a \in A .
$$

Proposition 1.7. ([8]). Let $(A, \mu)$ be a G-partially crossed P-module, the groups $G$ and $P$ acting on each other and on $A$ compatibly.

Under the aforementioned actions of $G$ and $P$ on $\operatorname{Der}(G,(A, \mu))$ and the homomorphism $\gamma: \operatorname{Der}(G,(A, \mu)) \rightarrow P$ given by $(\alpha, r) \rightarrow r$, the pair $(\operatorname{Der}(G,(A, \mu)), \gamma)$ is a $G$-precrossed P-module.

2. The first non-abelian cohomology. Let $(A, \mu)$ be a $G$-partially crossed $P$-module. Define on the group $\operatorname{Der}(G,(A, \mu))$ an equivalence relation as follows:

$$
(\alpha, r) \sim(\beta, s) \Longleftrightarrow\left\{\begin{array}{l}
\text { there exists } a \in A: \beta(x)=a^{-1} \alpha(x){ }^{x} a, \\
s=\mu(a)^{-1} r \bmod H^{0}(G, P) .
\end{array}\right.
$$

TheORem 2.1. Let $(A, \mu)$ be a $G$-partially crossed P-module satisfying the following conditions: 
(1) $H^{0}(G, P)$ is a normal subgroup of $P$,

(2) for any $c \in H^{0}(G, P)$ and $(\alpha, r) \in \operatorname{Der}(G,(A, \mu))$ there exists $a \in A$ such that $\mu(a)=1$ and ${ }^{c} \alpha(x)=a^{-1} \alpha(x)^{x} a, x \in G$.

Then the group $\operatorname{Der}(G,(A, \mu))$ induces on the quotient set $\operatorname{Der}(G,(A, \mu)) / \sim a$ group structure and this quotient group will be called the first cohomology group $H^{1}(G,(A, \mu))$ of the group $G$ with coefficients in the G-partially crossed P-module $(A, \mu)$.

Proof. We have to show that the relation $\sim$ is a congruence, that is if $(\alpha, r) \sim\left(\alpha^{\prime}, r^{\prime}\right)$ and $(\beta, s) \sim\left(\beta^{\prime}, s^{\prime}\right)$, then $(\alpha, r)(\beta, s) \sim\left(\alpha^{\prime}, r^{\prime}\right)\left(\beta^{\prime}, s^{\prime}\right)$. For this we will use Guin's proof [6], which remains valid in our generalized case.

We first prove that

$$
(\alpha, r)(\beta, s) \sim(\alpha, r c)(\beta, s)
$$

for any $c \in H^{0}(G, P)$. For $(\beta, s)$ and $c \in H^{0}(G, P)$ there is $a \in A$ such that $\mu(a)=1$ and $a^{-1} \beta(x)^{x} a, x \in G$. One gets

$$
\begin{aligned}
{ }^{r c} \beta(x) \alpha(x) & ={ }^{r}\left(a^{-1} \beta(x){ }^{x} a\right) \alpha(x)={ }^{r} a^{-1}{ }^{r} \beta(x){ }^{r x} a \alpha(x) \\
& ={ }^{r} a^{-1}{ }^{r} \beta(x) \alpha(x){ }^{x r} a .
\end{aligned}
$$

Since $\mu\left({ }^{r} a\right)^{-1}=\left(r \mu(a) r^{-1}\right)^{-1}=1$, one has $r c s=\mu\left({ }^{r} a\right)^{-1} r s c^{\prime}$ with $c^{\prime} \in H^{0}(G, P)$. Therefore, $(\alpha, r)(\beta, s)=(\alpha, r c)(\beta, s)$.

We have equalities

$$
\alpha^{\prime}(x)=b^{-1} \alpha(x){ }^{x} b, \quad r^{\prime}=\mu(b)^{-1} r z
$$

and

$$
\beta^{\prime}(x)=d^{-1} \beta(x){ }^{x} d, \quad s^{\prime}=\mu(d)^{-1} s t
$$

with $z, t \in H^{0}(G, P)$. Set

$$
(\alpha, r z)(\beta, s)=(\gamma, r z s) \text { and }\left(\alpha^{\prime}, r^{\prime}\right)\left(\beta^{\prime}, s^{\prime}\right)=\left(\gamma^{\prime}, r^{\prime} s^{\prime}\right)
$$

with $\gamma(x)={ }^{r z} \beta(x) \alpha(x)$ and $\gamma^{\prime}(x)={ }^{r^{\prime}} \beta^{\prime}(x) \alpha^{\prime}(x), x \in G$. It will be shown that

$$
(\alpha, r z)(\beta, s) \sim\left(\alpha^{\prime}, r^{\prime}\right)\left(\beta^{\prime}, s^{\prime}\right) .
$$

Indeed,

$$
\begin{aligned}
\gamma^{\prime}(x) & ={ }^{r^{\prime}}\left(d^{-1} \beta(x)^{x} d\right) b^{-1} \alpha(x)^{x} b \\
& ={ }^{\mu(b)^{-1} r z} d^{-1 \mu(b)^{-1} r z} \beta(x)^{\mu(b)^{-1} r z x} d b^{-1} \alpha(x)^{x} b \\
& =b^{-1 r z} d^{-1 r z} \beta(x)^{r x}\left({ }^{z} d\right) \alpha(x)^{x} b \\
& =b^{-1 r z} d^{-1 r z} \beta(x) \alpha(x)^{x r z} d^{x} b,
\end{aligned}
$$

and

$$
\begin{gathered}
\mu\left({ }^{r z} d b^{-1}\right)=\mu(b)^{-1} r z \mu(d)^{-1} z^{-1} r^{-1}=r^{\prime} s^{\prime} t^{-1} s^{-1} z^{-1} r^{-1}, \\
r^{\prime} s^{\prime}=\mu\left({ }^{r z} d b\right)^{-1} r z s t
\end{gathered}
$$

with $t \in H^{0}(G, P)$. 
It follows that $(\alpha, r z)(\beta, s) \sim\left(\alpha^{\prime}, r^{\prime}\right)\left(\beta^{\prime}, s^{\prime}\right)$. Therefore $(\alpha, r)(\beta, s) \sim\left(\alpha^{\prime}, r^{\prime}\right)\left(\beta^{\prime}, s^{\prime}\right)$ and the equivalence $\sim$ is a congruence.

Clearly any partially crossed $G$-module viewed as a $G$-partially crossed $G$-module satisfies conditions of Theorem 2.1. In this case $H^{0}(G, G)=Z(G)$ and for $(\alpha, g) \in \operatorname{Der}(G,(A, \mu))=\operatorname{Der}_{G}(G, A), c \in Z(G)$ the equality $\alpha(c x)=\alpha(x c), x \in G$, implies $\alpha(c){ }^{c} \alpha\left((x)=\alpha(x){ }^{x} \alpha(c)\right.$ and $\mu(\alpha(c))=g c g^{-1} c^{-1}=1$. We recover Guin's first cohomology group of a group $G$ with coefficients in a crossed $G$-module [6].

If $f:(A, \mu) \rightarrow(B, \lambda)$ is a homomorphism of $G$-partially crossed $P$-modules satisfying the conditions of Theorem 2.1, then $f^{*}$ induces a homomorphism $f^{1}: H^{1}(G,(A, \mu)) \rightarrow H^{1}(G,(B, \lambda))$. The above defined action of $G$ on $\operatorname{Der}(G,(A, \mu))$ induces an action of $G$ on $H^{1}(G,(A, \mu))$ given by

$$
{ }^{g}[(\alpha, r)]=\left[{ }^{g}(\alpha, r)\right], \quad g \in G .
$$

In our next statement it will be shown that Guin's first non-abelian cohomology group is closely related with torsors. A similar relationship between the first nonabelian pointed set cohomology and principal homogeneous spaces is well-known [1]. To this end, the notion of a $G$-torsor over a partially crossed $G$-module will be introduced.

Definition 2.2. A $G$-torsor over a partially crossed $G$-module $(A, \mu)$ is a pair $(E, f)$ consisting of a non-empty $G$-set $E$ with an action on the right of $A$ on $E$ denoted by $x a$ (for $x \in E, a \in A$ ) which is compatible with the action of $G$ and such that for any $x, y \in E$ there is a unique element $b \in A$ with $y=x b$, and $f$ is a map from $E$ to $G$ such that

(1) for any $x \in E, s \in G$ the following equality holds

$$
\mu(a)=f(x) s f(x)^{-1} s^{-1}
$$

with ${ }^{s} x=x a, a \in A$;

(2) if $y=x b$ then

$$
f(y)=\mu\left(b^{-1}\right) f(x), \quad x, y \in E, b \in A .
$$

Definition 2.3. It will be said that $G$-torsors $(E, f)$ and $\left(E^{\prime}, f^{\prime}\right)$ over a partially crossed $G$-module $(A, \mu)$ are isomorphic if there is a bijection $\vartheta: E \rightarrow E^{\prime}$ compatible with the actions of $G$ and $A$ such that

$$
f(x)=f^{\prime} \vartheta(x) \bmod Z(G)
$$

for any $x \in E$.

Denote by $E(G, A)$ the set of classes of isomorphic $G$-torsors over the partially crossed $G$-module $(A, \mu)$.

A product on the set $E(G, A)$ is introduced as follows. Let $\left[\left(E_{1}, f_{1}\right)\right]$, $\left[\left(E_{2}, f_{2}\right)\right] \in E(G, A)$ and let $x \in E_{1}, y \in E_{2}$. Take $A$ with a new action of $G$ given by $\left({ }^{s} a\right)^{\prime}={ }^{f_{1}(x)} c b^{s} a$ for any $s \in G, a \in A$ with ${ }^{s} x=x b,{ }^{s} y=y c$. Denote this $G$-group by $E$ and define the action of $A$ on $E$ by translation on the right. Define a map $g: E \rightarrow G$ given by 


$$
g(a)=\mu\left(a^{-1}\right) f_{1}(x) f_{2}(x) .
$$

Then the pair $(E, g)$ is a $G$-torsor over the partially crossed $G$-module $(A, \mu)$. Define the product by

$$
\left[\left(E_{1}, f_{1}\right)\right] \circ\left[\left(E_{2}, f_{2}\right)\right]=[(E, g)] .
$$

Theorem 2.4. If $(A, \mu)$ is a partially crossed G-module, there is a natural isomorphism between $E(G, A)$ and $H^{1}(G, A)$.

Proof. Let $[(E, f)] \in E(G, A)$ and take $x \in E$. For any $s \in G$ one has ${ }^{s} x=x a$ and an induced map $\alpha_{x}: G \longrightarrow A$ defined by $\alpha_{x}(s)=a$ that is a cocycle. Using 1) of Definition 2.2 we see that the pair $\left(\alpha_{x}, f(x)\right)$ is an element of $\operatorname{Der}(G,(G, A))=\operatorname{Der}_{G}(G, A)$.

Define a map $\alpha: E(G, A) \longrightarrow H^{1}(G, A)$ by $\alpha([(E, f)])=\left[\left(\alpha_{x}, f(x)\right)\right]$. We have to show that $\alpha$ is well-defined.

If $y \in E$ and $y=x b, b \in A$, then ${ }^{s} y={ }^{s} x{ }^{s} b=x a{ }^{s} b=x b b^{-1} a^{s} b=y b^{-1} a^{s} b$. By 2) $f(y)=\mu\left(b^{-1}\right) f(x)$. It follows that $\left(\alpha_{x}, f(x)\right) \sim\left(\alpha_{y}, f(y)\right)$. Let $(E, f)$ be isomorphic to $\left(E^{\prime}, f^{\prime}\right)$, that is there is a bijection $\vartheta: E \longrightarrow E^{\prime}$ with properties given in Definition 2.2. Take $x^{\prime} \in E^{\prime}$ and $\vartheta(x)=x^{\prime}, x \in E$. Then $\vartheta\left({ }^{s} x\right)={ }^{s} \vartheta(x)={ }^{s} x^{\prime}$ and $\vartheta(x a)=\vartheta(x) a=$ $x^{\prime} a$ with ${ }^{s} x=x a$. Thus, $\alpha_{x}=\alpha_{x^{\prime}}$. Since $f(x)=f^{\prime}\left(x^{\prime}\right) \bmod Z(G)$, one deduces $\left(\alpha_{x}, f(x)\right) \sim\left(\alpha_{x^{\prime}}, f^{\prime}\left(\left(x^{\prime}\right)\right)\right.$. Therefore, the map $\alpha$ is well-defined.

Let $[(\alpha, g)] \in H^{1}(G, A)$. Take $A$ with a new action of $G$ given by $\left({ }^{s} x\right)^{\prime}=\alpha(s){ }^{s} x$, $x \in A, s \in G$, and with the action of $A$ on itself by translation on the right. Denote this $G$-set by $P_{\alpha}$. Define a map $f_{g}: P_{\alpha} \rightarrow G$ by $f_{g}(x)=\mu\left(x^{-1}\right) g, x \in P_{\alpha}$, which verifies conditions 1) and 2) of Definition 2.2. In effect, if $\left({ }^{s} x\right)^{\prime}=x a$ then $\alpha(s)^{s} x=x a$. On the other hand $\mu \alpha(s)=g s g^{-1} s^{-1}$. Thus, $\mu \alpha(s) \mu\left({ }^{s} x\right)=\mu(x a), g s g^{-1} s^{-1} s \mu(x) s^{-1}=$ $\mu(x) \mu(a)$. Whence

$$
\mu(a)=\mu(x)^{-1} g s g^{-1} \mu(x) s^{-1}=f_{g}(x) s f_{g}(x)^{-1} s^{-1}
$$

and $f_{g}$ verifies condition 1 ). If $y=x b$ then one has

$$
f_{g}(y)=\mu\left(y^{-1}\right) g=\mu\left(b^{-1} x^{-1}\right) g=\mu\left(b^{-1}\right) \mu\left(x^{-1}\right) g=\mu\left(b^{-1}\right) f(x) .
$$

Thus $f_{g}$ satisfies condition 2) too. One gets a $G$-torsor $\left(P_{\alpha}, f_{g}\right)$ over the partially crossed $G$-module $(A, \mu)$ and define $\beta: H^{1}(G, A) \rightarrow E(G, A)$ by $\beta([(\alpha, g)])=$ $\left[\left(P_{\alpha}, f_{g}\right)\right]$.

If $(\alpha, g) \sim\left(\alpha^{\prime}, g^{\prime}\right)$ then $\alpha^{\prime}(s)=b^{-1} \alpha(s)^{s} b$ and $g^{\prime}=\mu\left(b^{-1}\right) g \bmod Z(G)$. We will show that $\left(P_{\alpha}, f_{g}\right)$ is isomorphic $\left(P_{\alpha^{\prime}}, f_{g^{\prime}}\right)$.

Define $\partial: P_{\alpha} \longrightarrow P_{\alpha^{\prime}}$ by $\partial(x)=b^{-1} x, x \in P_{\alpha}$. Then $\partial\left(\left({ }^{s} x\right)^{\prime}\right)=b^{-1} \alpha(s){ }^{s} x$ and $\left({ }^{s}(\partial(x))\right)^{\prime}=\left({ }^{s}\left(b^{-1} x\right)\right)^{\prime}=\alpha^{\prime}(s){ }^{s}\left(b^{-1} x\right)=b^{-1} \alpha(s){ }^{s} b^{s} b^{-1}{ }^{s} x=b^{-1} \alpha(s){ }^{s} x$. Thus, $\partial\left(\left({ }^{s} x\right)^{\prime}\right)=$ $\left({ }^{s}(\partial(x))\right)^{\prime}$. It is obvious that $\partial$ preserves the action of $A$.

For $x \in P_{\alpha}$ one gets

$$
\begin{aligned}
\left(f_{g}^{\prime}(\partial(x))=f_{g}^{\prime}\left(b^{-1} x\right)=\mu\left(x^{-1} b\right) g^{\prime}\right. & =\mu\left(x^{-1}\right) \mu(b) \mu\left(b^{-1}\right) g \bmod Z(G) \\
& =\mu\left(x^{-1}\right) g \bmod Z(G) \\
& =f_{g}(x) \bmod Z(G) .
\end{aligned}
$$


This implies that $\left(P_{\alpha}, f_{g}\right)$ is isomorphic to $\left(P_{\alpha^{\prime}}, f_{g^{\prime}}\right)$. Therefore the map $\beta$ is welldefined.

It is easily checked that $\alpha$ is a homomorphism and $\alpha \beta=1, \beta \alpha=1$.

3. The second non-abelian cohomology. Now the second cohomology $H^{2}(G,(A, \mu))$ of a group $G$ with coefficients in a $G$-partially crossed $P$-module $(A, \mu)$ will be defined. Consider the diagram

$$
M \underset{l_{1}}{\stackrel{l_{0}}{\rightarrow}} F \stackrel{\tau}{\rightarrow} G
$$

with $F$ a free group, $\tau$ is a surjective homomorphism, $M$ the group consisting of pairs $(x, y), x, y \in F$, such that $\tau(x)=\tau(y)$ and $l_{0}, l_{1}$ are canonical projections, $l_{0}(x, y)=x$, $l_{1}(x, y)=y$. This means $\left(M, l_{0}, l_{1}\right)$ is the simplicial kernel of $\tau$. Put $\Delta=\{(x, x)$, $x \in F\} \subset M$.

Then $(A, \mu)$ can be viewed as a $F$-partially crossed $P$-module induced by $\tau$ and as a $M$-partially crossed $P$-module induced by $\tau l_{0}$ (or by $\tau l_{1}$ ). Let $\widetilde{Z}^{1}(M,(A, \mu)$ ) be the subset of $\operatorname{Der}(M,(A, \mu))$ consisting of elements of the form $(\alpha, 1)$ satisfying the condition $\alpha(\Delta)=1$, implying $\alpha(M) \subset Z(A)$. It follows that $\widetilde{Z}^{1}(M,(A, \mu))$ is an abelian subgroup of $\operatorname{Der}(M,(A, \mu))$.

Define on $\widetilde{Z}^{1}(M,(A, \mu))$ a relation by

$$
\left(\alpha^{\prime}, 1\right) \sim(\alpha, 1) \Longleftrightarrow(\beta, h) \in \operatorname{Der}(F,(A, \mu))
$$

such that the following equality holds

$$
\left(\alpha^{\prime}, 1\right)=\left(\beta l_{0}, h\right)(\alpha, 1)\left(\beta l_{1}, h\right)^{-1}
$$

in the group $\operatorname{Der}(M,(A, \mu))$.

We see that if $\left(\alpha^{\prime}, 1\right) \sim(\alpha, 1)$ one has

$$
\alpha^{\prime}(x)=\beta l_{1}(x)^{-1 h} \alpha(x) \beta l_{0}(x), \quad x \in M .
$$

Proposition 3.1. The relation $\sim$ defined on $\widetilde{Z}^{1}(M,(A, \mu))$ is an equivalence.

Proof. Clearly this relation is reflexive. If $\left(\alpha^{\prime}, 1\right) \sim(\alpha, 1)$, that is $\left(\alpha^{\prime}, 1\right)=\left(\beta l_{0}, h\right)(\alpha, 1)\left(\beta l_{1}, 1\right)^{-1}$ with $(\beta, h) \in \operatorname{Der}(F,(A, \mu))$, then $(\alpha, 1)=\left(\beta l_{0}, h\right)^{-1}$. $\cdot\left(\alpha^{\prime}, 1\right)\left(\beta l_{1}, h\right)$ and $\left(\beta l_{0}, h\right)^{-1}=\left(\widetilde{\beta} l_{0}, h^{-1}\right), \quad\left(\beta l_{1}, h\right)=\left(\widetilde{\beta} l_{1}, h^{-1}\right)^{-1}$ with $\left(\widetilde{\beta}, h^{-1}\right)=$ $(\beta, h)^{-1} \in \operatorname{Der}(F,(A, \mu))$. Thus the relation $\sim$ is symmetric. It remains to show transitivity.

Let $\left(\alpha^{\prime}, 1\right) \sim(\alpha, 1)$ and $\left(\alpha^{\prime \prime}, 1\right) \sim\left(\alpha^{\prime}, 1\right)$. Then one has

$$
\begin{gathered}
\left(\alpha^{\prime}, 1\right)=\left(\beta l_{0}, h\right)(\alpha, 1)\left(\beta l_{1}, h\right)^{-1}, \\
\left(\alpha^{\prime \prime}, 1\right)=\left(\beta^{\prime} l_{0}, h^{\prime}\right)\left(\alpha^{\prime}, 1\right)\left(\beta^{\prime} l_{1}, h^{\prime}\right)^{-1}
\end{gathered}
$$

with $(\beta, h),\left(\beta^{\prime}, h^{\prime}\right) \in \operatorname{Der}(F,(A, \mu))$. 
It follows that

$$
\begin{aligned}
\left(\alpha^{\prime \prime}, 1\right) & =\left(\beta^{\prime} l_{0}, h^{\prime}\right)\left(\beta l_{0}, h\right)(\alpha, 1)\left(\beta l_{1}, h\right)^{-} 1\left(\beta^{\prime} l_{1}, h^{\prime}\right)^{-1} \\
& =\left(\left(\beta^{\prime *} \beta\right) l_{0}, h^{\prime} h\right)(\alpha, 1)\left(\left(\beta^{\prime *} \beta\right) l_{1}, h^{\prime} h\right)^{-1}
\end{aligned}
$$

with $\left(\beta^{\prime} * \beta, h^{\prime} h\right)=\left(\beta^{\prime}, h^{\prime}\right)(\beta, h) \in \operatorname{Der}(F,(A, \mu))$. Therefore $\left(\alpha^{\prime \prime}, 1\right) \sim(\alpha, 1)$ and the relation $\sim$ is an equivalence.

Proposition 3.2. The quotient set $\widetilde{Z}^{1}(M,(A, \mu)) / \sim$ is independent of the diagram (3.1) and is unique up to bijection.

We need the following.

Lemma 3.3. Let $A$ be a $G$-group and let $\alpha: M \rightarrow A$ be a crossed homomorphism such that $\alpha(\Delta)=1$. Then there exists a map $q: F \rightarrow A$ such that for $y \in M$

$$
\alpha(y)=q l_{1}(y)^{-1} q l_{0}(y) .
$$

Proof. Note that if $\left(x, x^{\prime \prime}\right),\left(x^{\prime}, x^{\prime \prime}\right) \in M$, then $\alpha\left(x, x^{\prime \prime}\right)=\alpha\left(x^{\prime}, x^{\prime \prime}\right) \cdot \alpha\left(x, x^{\prime}\right)$. Indeed, the equalities $\left(x, x^{\prime \prime}\right)=\left(1, x^{\prime \prime} x^{\prime-1}\right)\left(x, x^{\prime}\right)$ and $\left(x^{\prime}, x^{\prime \prime}\right)=\left(1, x^{\prime \prime} x^{\prime-1}\right)\left(x^{\prime}, x^{\prime}\right)$ imply $\quad \alpha\left(x, x^{\prime \prime}\right)=\alpha\left(1, x^{\prime \prime} x^{\prime-1}\right) \alpha\left(x, x^{\prime}\right) \quad$ and $\quad \alpha\left(x^{\prime}, x^{\prime \prime}\right)=\alpha\left(1, x^{\prime \prime} x^{\prime-1}\right) \alpha\left(x^{\prime}, x^{\prime}\right)=$ $\alpha\left(1, x^{\prime \prime} x^{\prime-1}\right)$, giving the required equality.

In particular, applying this equality for $(x, x),\left(x^{\prime}, x\right) \in M$ one gets $\alpha(x, x)=$ $\alpha\left(x^{\prime}, x\right) \alpha\left(x, x^{\prime}\right)$. Therefore $\alpha\left(x^{\prime}, x\right)=\alpha\left(x, x^{\prime}\right)^{-1}$ for any $\left(x, x^{\prime}\right) \in M$.

Take a section $\eta: G \longrightarrow F, \tau \eta=1_{G}$ and define a map $q: F \longrightarrow A$ by

$$
q(x)=\alpha(x, \eta \tau(x)), \quad x \in F .
$$

For $\left(x, x^{\prime}\right) \in M$ one has

$$
\begin{aligned}
q l_{1}\left(x, x^{\prime}\right)^{-1} q l_{0}\left(x, x^{\prime}\right) & =q\left(x^{\prime}\right)^{-1} q(x) \\
& =\left(\alpha\left(x^{\prime}, \eta \tau\left(x^{\prime}\right)\right)^{-1} \alpha(x, \eta \tau(x))\right. \\
& =\alpha\left(\eta \tau\left(x^{\prime}\right), x^{\prime}\right) \alpha(x, \eta \tau(x)) .
\end{aligned}
$$

On the other hand, since $\alpha\left(x, x^{\prime}\right)=\alpha\left(1, x^{\prime} x^{-1}\right)$ for all $\left(x, x^{\prime}\right) \in M$, one gets $\alpha\left(\eta \tau\left(x^{\prime}\right), x^{\prime}\right)=\alpha\left(1, x^{\prime} \eta \tau\left(x^{\prime}\right)^{-1}\right)$ and $\alpha(x, \eta \tau(x))=\alpha\left(1, \eta \tau(x) x^{-1}\right)$. But $\left(1, x^{\prime} \eta \tau\left(x^{\prime}\right)^{-1}\right)$ $\left(1, \eta \tau(x) x^{-1}\right)=\left(1, x^{\prime} x^{-1}\right)$. Therefore, we obtain the equality

$$
\alpha\left(x, x^{\prime}\right)=\alpha\left(1, x^{\prime} \eta \tau\left(x^{\prime}\right)^{-1}\right) \alpha\left(1, \eta \tau(x) x^{-1}\right)=q l_{1}\left(x, x^{\prime}\right)^{-1} q l_{0}\left(x, x^{\prime}\right) .
$$

Proof of Proposition 3.2. Consider the commutative diagram

$$
\begin{array}{ccccc}
M^{\prime} & \stackrel{l_{0}^{\prime}}{\rightarrow} & F^{\prime} & \stackrel{\tau^{\prime}}{\rightarrow} & G \\
\overline{\gamma_{1}} \downarrow \overline{\gamma_{2}} & \stackrel{l_{1}^{\prime}}{\rightarrow} & \gamma_{1} \downarrow \gamma_{2} & & \| \\
M & \stackrel{l_{0}}{\rightarrow} & F & \stackrel{\tau}{\rightarrow} & G,
\end{array}
$$


$\left(M, l_{0}, l_{1}\right)$ and $\left(M^{\prime}, l_{0}^{\prime}, l_{1}^{\prime}\right)$ being the simplicial kernels of $\tau$ and $\tau^{\prime}$ respectively, $l_{i} \overline{\gamma_{1}}=\gamma_{1} l_{i}^{\prime}, l_{i} \overline{\gamma_{2}}=\gamma_{2} l_{i}^{\prime}, i=0,1, \tau \gamma_{1}=\tau \gamma_{2}=\tau^{\prime}$.

The pair $\left(\gamma_{i}, \overline{\gamma_{i}}\right)$ induces a homomorphism

$$
\operatorname{Der}(M,(A, \mu)) \longrightarrow \operatorname{Der}\left(M^{\prime},(A, \mu)\right)
$$

given by $(\alpha, r) \rightarrow\left(\alpha \overline{\gamma_{i}}, r\right), i=1,2$.

If $\left(\alpha^{\prime}, 1\right) \sim(\alpha, 1), \quad$ that $\quad$ is $\quad\left(\alpha^{\prime}, 1\right)=\left(\beta l_{0}, h\right)(\alpha, 1)\left(\beta l_{1}, h\right)^{-1} \quad$ with $\quad(\beta, h) \in$ $\operatorname{Der}(F,(A, \mu))$, then

$$
\alpha^{\prime} \overline{\gamma_{i}}(y)=\beta \gamma_{i} l_{1}^{\prime}(y)^{-1}{ }^{h} \alpha \overline{\gamma_{i}}(y) \beta \gamma_{i} l_{0}^{\prime}(y), \quad y \in M^{\prime} .
$$

Thus $\left(\alpha^{\prime} \overline{\gamma_{i}}, 1\right) \sim\left(\alpha \overline{\gamma_{i}}, 1\right), i=1,2$, and one gets a natural map

$$
\varepsilon_{i}: \widetilde{Z^{1}}(M,(A, \mu)) / \sim \longrightarrow \widetilde{Z}^{1}\left(M^{\prime},(A, \mu)\right) / \sim
$$

induced by the pair $\left(\gamma_{i}, \tilde{\gamma}_{i}\right)$ and given by $[(\alpha, 1)] \rightarrow\left[\left(\alpha \tilde{\gamma}_{i}, 1\right)\right], i=1,2$.

We will show that $\varepsilon_{1}=\varepsilon_{2}$. By Lemma 3.3 there is a map $q: F \rightarrow A$ such that

$$
\alpha(y)=q l_{1}(y)^{-1} q l_{0}(y), \quad y \in M .
$$

Take the homomorphism $s: F^{\prime} \rightarrow M$ given by

$$
s\left(x^{\prime}\right)=\left(\gamma_{1}\left(x^{\prime}\right), \gamma_{2}\left(x^{\prime}\right)\right), \quad x^{\prime} \in F^{\prime} .
$$

It is clear that $(\alpha s, 1) \in \operatorname{Der}\left(F^{\prime},(A, \mu)\right)$. Further one has

$$
\begin{aligned}
\left(\left(\alpha s l_{1}^{\prime}\right)^{-1} \alpha \tilde{\gamma}_{2} \alpha s l_{0}^{\prime}\right)\left(x_{0}^{\prime}, x_{1}^{\prime}\right) & =\alpha s\left(\left(x^{\prime}\right)^{-1} \alpha \tilde{\gamma}_{2}\left(x_{0}^{\prime}, x_{1}^{\prime}\right) \alpha s\left(x_{0}^{\prime}\right)\right. \\
& =\alpha\left(\gamma_{1}\left(x_{1}^{\prime}\right), \gamma_{2}\left(x_{1}^{\prime}\right)\right)^{-1} \alpha \tilde{\gamma}_{2}\left(x_{0}^{\prime}, x_{1}^{\prime}\right) \alpha\left(\gamma_{1}\left(x_{0}^{\prime}\right), \gamma_{2}\left(x_{0}^{\prime}\right)\right) \\
& =q \gamma_{1}\left(x_{1}^{\prime}\right)^{-1} q \gamma_{2}\left(x_{1}^{\prime}\right) \\
& =q \gamma_{2}\left(x_{1}^{\prime}\right)^{-1} q \gamma_{2}\left(x_{0}^{\prime}\right) q \gamma_{2}\left(x_{0}^{\prime}\right)^{-1} q \gamma_{1}\left(x_{0}^{\prime}\right) \\
& =q \gamma_{1}\left(x_{1}^{\prime}\right)^{-1} q \gamma_{1}\left(x_{0}^{\prime}\right) \\
& =\alpha \tilde{\gamma}_{1}\left(x_{0}^{\prime}, x_{1}^{\prime}\right)
\end{aligned}
$$

for any $\left(x_{0}^{\prime}, x_{1}^{\prime}\right) \in M^{\prime}$. Therefore $\left(\alpha \tilde{\gamma_{1}}, 1\right) \sim\left(\alpha \tilde{\gamma_{2}}, 1\right)$ with $(\alpha s, 1) \in \operatorname{Der}\left(F^{\prime},(A, \mu)\right)$, implying the required equality $\varepsilon_{1}=\varepsilon_{2}$.

The proof of the uniqueness is standard.

It is easy to check that the quotient set $\widetilde{Z}^{1}(M,(A, \mu)) / \sim$ is naturally bijective to $H^{2}(G, A)$ when $A$ is a $G$-module viewed as a crossed $G$-module. That fact motivates the following

Definition 3.4. Let $(A, \mu)$ be a $G$-partially crossed $P$-module. The quotient set $\widetilde{Z^{1}}(M,(A, \mu)) / \sim$ will be called the second cohomology of $G$ with coefficients in $(A, \mu)$ and denoted by $H^{2}(G,(A, \mu))$.

A homomorphism of $G$-partially crossed $P$-modules $f:(A, \mu) \rightarrow(B, \lambda)$ induces a map of pointed sets 


$$
f^{2}: H^{2}(G,(A, \mu)) \longrightarrow H^{2}(G,(B, \lambda))
$$

given by $f^{2}([(\alpha, 1)])=[(f \alpha, 1)]$. It is easy to see that there is a canonical surjective $\operatorname{map} \vartheta: H^{2}(G, \operatorname{Ker} \mu) \rightarrow H^{2}(G,(A, \mu))$ given by $[\alpha] \mapsto[(\alpha, 1)]$.

Proposition 3.5. Let $(A, \mu)$ be a G-partially crossed P-module. There is an action of $G$ on $H^{2}(G,(A, \mu))$ such that $Z(G)$ acts trivially. If $P$ acts on $G$ and satisfies the compatibility condition (1.4), then there is also an action of $P$ on $H^{2}(G,(A, \mu))$.

Proof. Consider the diagram

$$
M_{G} \underset{l_{1}}{\stackrel{l_{0}}{\rightarrow}} F_{G} \stackrel{\tau_{G}}{\rightarrow} G
$$

with $F_{G}$ the free group generated by $G, \tau_{G}$ is the canonical homomorphism and $\left(M_{G}, l_{0}, l_{1}\right)$ is the simplicial kernel of $\tau_{G}$. There is an action of $G$ on $F_{G}$ defined as follows:

$$
{ }^{g}\left(\left|g_{1}\right|^{\varepsilon} \cdots\left|g_{n}\right|^{\varepsilon}\right)=\left|{ }^{g} g_{1}\right|^{\varepsilon} \ldots\left|{ }^{g} g_{n}\right|^{\varepsilon}, \quad g, g_{1}, \ldots, g_{n} \in G
$$

with $\varepsilon= \pm 1$. This action induces an action of $G$ on $M_{G}$ by

$$
{ }^{g}\left(x, x^{\prime}\right)=\left({ }^{g} x,{ }^{g} x^{\prime}\right), \quad g \in G, \quad\left(x, x^{\prime}\right) \in M_{G} .
$$

Finally one gets an action of $G$ on $\operatorname{Der}\left(M_{G},(A, \mu)\right)$ given by

$$
{ }^{g}(\alpha, r)=\left(\widetilde{\alpha},{ }^{g} r\right)
$$

with $\widetilde{\alpha}(m)={ }^{g} \alpha\left(g^{-1} m\right), g \in G, m \in M_{G}$, inducing an action of $G$ on $\widetilde{\operatorname{Der}}\left(M_{G},(A, \mu)\right)$ and on $\widetilde{Z}^{1}\left(M_{G},(A, \mu)\right)$ too. If $(\alpha, 1) \sim\left(\alpha^{\prime}, 1\right)$ it is easy to see that ${ }^{g}(\alpha, 1) \sim^{g}\left(\alpha^{\prime}, 1\right)$, $g \in G$, defining an action of $G$ on $H^{2}(G,(A, \mu))$. Since the above defined surjective map $\vartheta: H^{2}(G, \operatorname{Ker} \mu) \rightarrow H^{2}(G,(A, \mu))$ is a $G$-map and $Z(G)$ acts trivially on $H^{2}(G, \operatorname{Ker} \mu)$, it follows that $Z(G)$ acts trivially on $H^{2}(G,(A, \mu))$ too.

Let $(A, \mu)$ be a $G$-partially crossed $P$-module. It can be shown easily that there is an action of $H^{0}(G, P)$ on $H^{2}(G, \operatorname{Ker} \mu)$ given by ${ }^{r}[\alpha]=\left[{ }^{r} \alpha\right], r \in H^{0}(G, P)$ with $\alpha: M_{G} \rightarrow \operatorname{Ker} \mu$ a crossed homomorphism under the action of $G$ on $A$ such that $\alpha(\Delta)=1$.

Let

$$
1 \longrightarrow(A, 1) \stackrel{\varphi}{\rightarrow}(B, \mu) \stackrel{\psi}{\rightarrow}(C, \lambda) \longrightarrow 1
$$

be a short exact sequence of $G$-partially crossed $P$-modules. If the action of $H^{0}(G, P)$ on $H^{2}(G, A)$ is trivial then there is an action of $H^{1}(G,(C, \lambda))$ on $H^{2}(G, A)$ given by

$$
{ }^{(\alpha, r)}[\gamma]=\left[{ }^{r} \gamma\right] .
$$

We have to show that ${ }^{r} \gamma$ is a crossed homomorphism and this action is well-defined. Consider the diagram 


$$
\begin{array}{lllll}
M_{G} & \stackrel{l_{0}}{\rightarrow} & F_{G} & \stackrel{\tau_{G}}{\rightarrow} & G \\
& & & & \downarrow^{\alpha} \\
A & \stackrel{\varphi}{\rightarrow} & B & \stackrel{\psi}{\rightarrow} & C
\end{array}
$$

There is a crossed homomorphism $\beta: F_{G} \rightarrow B$ such that $\psi \beta=\alpha \tau_{G}$. Take the product

$$
\left(\beta l_{0}, r\right)(\varphi \gamma, 1)\left(\beta l_{0}, r\right)^{-1}=(\tilde{\gamma}, 1)
$$

in the group $\operatorname{Der}\left(M_{G},(B, \mu)\right)$. Then $\tilde{\gamma}(x)=\beta(x)^{-1 r} \varphi \gamma(x) \beta(x)={ }^{r} \varphi \gamma(x), x \in M_{G}$. Therefore ${ }^{r} \gamma: M_{G} \rightarrow A$ is a crossed homomorphism such that ${ }^{r} \gamma(\Delta)=1$. If $\left(\alpha^{\prime}, r^{\prime}\right) \in$ $[(\alpha, r)] \in H^{1}(G,(C, \lambda))$, that is $(\alpha, r) \sim\left(\alpha^{\prime}, r^{\prime}\right)$, then $\alpha^{\prime}(x)=c^{-1} \alpha(x)^{x} c$ and $r^{\prime}=\lambda(c)^{-1} r t$ with $c \in C, t \in H^{0}(G, P)$. It follows that

$$
\begin{aligned}
\varphi\left({ }^{r^{\prime}} \gamma(x)\right) & ={ }^{r^{\prime}} \varphi \gamma(x)={ }^{\lambda(c)^{-1} r t} \varphi \gamma(x) \\
& ={ }^{\mu(b)^{-1} r t} \varphi \gamma(x)=b^{-1}{ }^{r t} \varphi \gamma(x) b={ }^{r t} \varphi \gamma(x) \\
& =\varphi\left({ }^{r t} \gamma(x)\right),
\end{aligned}
$$

for $x \in M_{G}$, with $\psi(b)=c$. Hence $\left[{ }^{\prime} \gamma\right]=\left[{ }^{r} \gamma\right]=\left[{ }^{r} \gamma\right]$ proving the well-definedness of the action.

Using diagram (3.3) for the short exact sequence (3.2) one defines as follows a connecting map

$$
\delta^{1}: H^{1}(G,(C, \lambda)) \longrightarrow H^{2}(G, A)
$$

which is a crossed homomorphism when $(G,(C, \lambda))$ verifies conditions of Theorem 2.1. For $[(\alpha, r)] \in H^{1}(G,(C, \lambda))$ take a crossed homomorphism $\beta: F_{G} \rightarrow B$ such that $\psi \beta=\alpha \tau_{G}$. Thus there is a crossed homomorphism $\gamma: M_{G} \rightarrow A$ such that $\varphi \gamma=\left(\beta l_{1}\right)^{-1} \beta l_{0}$. It is clear that $\gamma(\Delta)=1$. Define

$$
\delta^{1}([(\alpha, r)])=[\gamma] .
$$

We must show the correctness of $\delta^{1}$. For another $\beta^{\prime}: F_{G} \rightarrow B$ with $\psi \beta^{\prime}=\alpha \tau_{G}$, one has $\psi \beta^{\prime}=\psi \beta$ and there is a crossed homomorphism $\sigma: F_{G} \rightarrow A$ such that $\beta^{\prime}=\beta \psi \sigma$. Then one gets

$$
\begin{aligned}
\varphi \gamma^{\prime} & =\left(\beta^{\prime} l_{1}\right)^{-1} \beta^{\prime} l_{0}=(\beta \varphi \sigma) l_{1}^{-1}(\beta \varphi \sigma) l_{0} \\
& =\varphi \sigma l_{1}^{-1} \beta l_{1}^{-1} \beta l_{0} \varphi \sigma l_{0}=\beta l_{1}^{-1} \beta l_{0} \varphi \sigma l_{1}^{-1} \varphi \sigma l_{0} \\
& =\varphi\left(\gamma \sigma l_{1}^{-1} \sigma l_{0}\right) .
\end{aligned}
$$

Hence $[\gamma]=\left[\gamma^{\prime}\right]$.

If $(\alpha, r) \sim\left(\alpha^{\prime}, r^{\prime}\right)$ then

$$
\begin{aligned}
\alpha^{\prime}(y) & =c^{-1} \alpha(y)^{y} c, c \in C, y \in M_{G}, \\
r^{\prime} & =\lambda(c)^{-1} r t, t \in H^{0}(G, P) .
\end{aligned}
$$


Take $\beta^{\prime}: F_{G} \rightarrow B$ such that $\beta^{\prime}(x)=b^{-1} \beta(x){ }^{x} b$ with $\psi(b)=c$ and $\psi \beta=\alpha \tau_{G}$. Then $\left(\beta^{\prime} l_{1}^{-1} \beta^{\prime} l_{0}\right)(y)=\beta^{\prime}\left(x_{2}\right)^{-1} \beta^{\prime}\left(x_{1}\right), y=\left(x_{1}, x_{2}\right) \in M_{G}$. Hence $\varphi \gamma^{\prime}(y)=\left(\beta^{\prime} l_{1}^{-1} \beta^{\prime} l_{0}\right)(y)=$ $\left(b^{-1} \beta\left(x_{2}\right){ }^{x_{2}} b\right)^{-1} b^{-1} \beta\left(x_{1}\right)^{x_{1}} b={ }^{x_{2}} b^{-1} \beta\left(x_{2}\right)^{-1} \beta\left(x_{1}\right)^{x_{1}} b=\beta\left(x_{2}\right)^{-1} \beta\left(x_{1}\right)=\varphi \gamma(y)$. Whence $\gamma^{\prime}=\gamma$. Therefore the connecting map $\delta^{1}$ is correctly defined.

For the short exact sequence 3.2 a connecting map $\delta^{2}: H^{2}(G,(C, \lambda)) \rightarrow$ $H^{3}(G, A)$ will be also defined. To this end consider the canonical free simplicial resolution of the group $G$ in the category of groups acting on the abelian group $A$ :

$$
\cdots \stackrel{\overrightarrow{!}}{\rightarrow} F_{3} \stackrel{\tau_{3}}{\rightarrow} M_{2} \underset{\frac{l_{3}^{2}}{\stackrel{l_{0}^{2}}{\rightarrow}}}{\vec{\longrightarrow}} F_{2} \stackrel{\tau_{2}}{\rightarrow} M_{1} \underset{l_{3}^{2}}{\stackrel{l_{0}^{2}}{\rightarrow}} F_{1} \stackrel{\tau_{1}}{\rightarrow} M_{0} \underset{l_{1}^{0}}{\stackrel{l_{0}^{0}}{\rightarrow}} F_{0} \stackrel{\tau_{0}}{\rightarrow} G
$$

where $F_{0}=F_{G}, \quad F_{i}=F_{M_{i-1}}, \quad i \geq 1, \quad \tau_{i}$ is the canonical homomorphism and $\left(M_{i}, l_{0}^{i}, \cdots, l_{i+1}^{i}\right)$ is the simplicial kernel of $\left(l_{0}^{i-1} \tau_{i}, \cdots, l_{i}^{i-1} \tau_{i}\right), i \geq 0$ (see [7]). We will use the equivalence of functors $H^{n+1}(-, A) \approx L_{n} \operatorname{Der}(-, A), n \geq 1$, when $A$ is a $Z[G]$ module. There is an action of $\operatorname{Der}\left(F_{0},(C, \lambda)\right)$ on $H^{3}(G, A)$ defined as follows:

$$
{ }^{(\alpha, r)}[f]=\left[{ }^{r} f\right],
$$

where $f: F_{2} \rightarrow A$ is a crossed homomorphism with $\prod_{i=0}^{3}\left(f l_{i}^{2} \tau_{3}\right)^{\varepsilon_{i}}=1, \varepsilon_{i}=(-1)^{i}$, and $(\alpha, r) \in \operatorname{Der}\left(F_{0},(C, \lambda)\right)$. The well-definedness of this action is proved similarly to the case of a short exact sequence of crossed $G$-modules (see [8]).

For any $G$-partially crossed $P$-module $(A, \mu)$ denote by $\operatorname{IDer}(G,(A, \mu))$ a subgroup of $\operatorname{Der}(G,(A, \mu))$ consisting of elements of the form $(\alpha, r), r \in H^{0}(G, P)$.

If either the aforementioned action of $\operatorname{Der}\left(F_{0},(C, \lambda)\right)$ on $H^{3}(G, A)$ is trivial or $\operatorname{Der}\left(F_{0},(C, \lambda)\right)=\operatorname{IDer}\left(F_{0},(C, \lambda)\right)$ and $H^{0}(G, P)$ acts trivially on $H^{2}(G$, Ker $\lambda)$, then there is a connecting map $\delta^{2}: H^{2}(G,(C, \lambda)) \rightarrow H^{3}(G, A)$ given by

$$
\delta^{2}([(\alpha, r)])=[\gamma], \quad(\alpha, 1) \in \widetilde{Z}^{1}\left(M_{G},(C, \lambda)\right),
$$

where $\varphi \gamma=\bar{\beta} \tau_{2}$ with $\bar{\beta}=\prod^{2}\left(\beta l_{i}^{1}\right)^{\varepsilon_{i}}, \varepsilon_{i}=(-1)^{i}$, and $\psi \beta=\alpha \tau_{1}$. The correctness of $\delta^{2}$ is proved similarly to the case of a short exact sequence of crossed $G$-modules (see [8]).

TheORem 3.6. Let (3.2) be a short exact sequence of G-partially crossed P-modules satisfying conditions of Theorem 2.1. Then there is an exact cohomology sequence

$$
\begin{aligned}
1 & \rightarrow H^{0}(G, A) \stackrel{\varphi^{0}}{\rightarrow} H^{0}(G, B) \stackrel{\psi^{0}}{\rightarrow} H^{0}(G, C) \stackrel{\delta^{0}}{\rightarrow} H^{1}(G, A) \stackrel{\varphi^{1}}{\rightarrow} H^{1}(G,(B, \mu)) \\
& \stackrel{\psi^{1}}{\rightarrow} H^{1}(G,(C, \lambda)) \stackrel{\delta^{1}}{\rightarrow} H^{2}(G, A) \stackrel{\varphi^{2}}{\rightarrow} H^{2}(G,(B, \mu)) \stackrel{\psi^{2}}{\rightarrow} H^{2}(G,(C, \lambda)),
\end{aligned}
$$

where $\varphi^{0}, \psi^{0}, \delta^{0}, \varphi^{1}$ and $\psi^{1}$ are homomorphisms. If in addition $H^{0}(G, P)$ acts trivially on $H^{2}(G, A)$, then $\delta^{1}$ is a crossed homomorphism under the action of $H^{1}(G,(C, \lambda))$ on $H^{2}(G, A)$ induced by the action of $P$ on $A$. Moreover, if either the action of $\operatorname{Der}\left(F_{0},(C, \lambda)\right)$ on $H^{3}(G, A)$ is trivial (in particular if $P$ acts trivially on $A$ ) or $\operatorname{Der}\left(F_{0},(C, \lambda)\right)=\operatorname{IDer}\left(F_{0},(C, \lambda)\right)$ and $H^{0}(G, P)$ acts trivially on $H^{2}(G$, Ker $\lambda)$, then the sequence

$$
H^{2}(G,(B, \mu)) \stackrel{\psi^{2}}{\rightarrow} H^{2}(G,(C, \lambda)) \stackrel{\delta^{2}}{\rightarrow} H^{3}(G, A)
$$

is also exact. 
Proof. The exactness of the sequence

$$
1 \rightarrow H^{0}(G, A) \stackrel{\varphi^{0}}{\rightarrow} H^{0}(G, B) \stackrel{\psi^{0}}{\rightarrow} H^{0}(G, C) \stackrel{\delta^{0}}{\rightarrow} H^{1}(G, A)
$$

is well known [10].

If $c \in H^{0}(G, C)$ then $\delta^{0}(c)=[\alpha]$ with $\alpha(x)=\varphi^{-1}\left(b^{-1}{ }^{x} b\right), x \in G$ and $\psi(b)=c$. It follows that $\left(\alpha_{0}, 1\right) \sim(\varphi \alpha, 1)$ where $\alpha_{0}$ is a trivial map, since $\varphi \alpha(x)=b^{-1} \alpha_{0}{ }^{x} b, x \in G$, and $\mu(b) \in H^{0}(G, P)$ because $\mu(b)=\lambda \psi(b)$ and ${ }^{x} \lambda(c)=\lambda\left({ }^{x} c\right)=\lambda(c), x \in G$. Therefore $\operatorname{Im} \delta^{0} \subset \operatorname{Ker} \varphi^{1}$.

Let $[\alpha] \in H^{1}(G, A)$ such that $\left(\alpha_{0}, 1\right) \sim(\varphi \alpha, 1)$. Then $\varphi \alpha(x)=b^{-1 x} b, x \in G$ and $\mu(b) \in H^{0}(G, P)$. One has $\psi\left(b^{-1}{ }^{x} b\right)=\psi \varphi \alpha(x)=1$. Thus $\psi(b)=\psi\left({ }^{x} b\right)={ }^{x} \psi(b)$, whence $\psi(b) \in H^{0}(G, C)$. Clearly $\delta^{0}(\psi(b))=[\alpha]$. Therefore $\operatorname{Ker} \varphi^{1} \subset \operatorname{Im} \delta^{0}$. Obviously the composite $\psi^{1} \varphi^{1}$ is the trivial map.

Let $[(\alpha, r)] \in H^{1}(G,(B, \mu))$ such that $\left(\alpha_{0}, 1\right) \sim(\psi \alpha, 1)$. Then $\psi \alpha(x)=c^{-1}{ }^{x} c$, $c \in C$, and $r=\lambda(c)^{-1} t, \quad t \in H^{0}(G, P)$. Let $\psi(b)=\lambda(c)$ and $r=\mu(b)^{-1} t$. Take $\widetilde{\alpha}(x)=b \alpha(x){ }^{x} b^{-1}, x \in G$. Since $\psi \widetilde{\alpha}(x)=1, x \in G$, one has $\varphi^{-1} \widetilde{\alpha}: G \rightarrow A$ and $(\alpha, r) \sim(\widetilde{\alpha}, 1)$. Therefore $\varphi^{1}\left(\left[\varphi^{-1} \widetilde{\alpha}\right]\right)=[(\alpha, r)]$.

Let $[(\alpha, r)] \in H^{1}(G,(B, \mu))$. Then $\psi^{1}([(\alpha, r)])=[(\psi \alpha, r)]$. Consider the diagram (3.3) and take the crossed homomorphism $\alpha \tau_{G}: F_{G} \rightarrow B$. Then $\varphi \gamma=\left(\alpha \tau_{G} l_{1}\right)^{-1} \alpha \tau_{G} l_{0}$ and $\delta^{1} \psi^{1}([(\alpha, r)])=[\gamma]$. But $\gamma=\alpha_{0}$ is the trivial map, since $\alpha \tau_{G} l_{0}=\alpha \tau_{G} l_{1}$. Therefore $\operatorname{Im} \psi^{1} \subset \operatorname{Ker} \delta^{1}$.

Let $[(\alpha, r)] \in H^{1}(G,(C, \lambda))$ such that $\delta^{1}([(\alpha, r)])=1$. If $\beta: F_{G} \rightarrow B$ is a crossed homomorphism such that $\psi \beta=\alpha \tau_{G}$, then $\delta^{1}([(\alpha, r)])=[\gamma]$ with $\varphi \gamma=\left(\beta l_{1}\right)^{-1} \beta l_{0}$. Thus there is a crossed homomorphism $\eta: F_{G} \rightarrow A$ such that $\gamma=\left(\eta l_{1}\right)^{-1} \eta l_{0}$. Hence one gets

$$
\left(\beta l_{1}\right)^{-1} \beta l_{0}=\left(\varphi \eta l_{1}\right)^{-1} \varphi \eta l_{0}, \quad\left(\varphi \eta^{-1} \beta\right) l_{0}=\left(\varphi \eta^{-1} \beta\right) l_{1},
$$

implying a crossed homomorphism $\bar{\alpha}: G \rightarrow B$ such that $(\varphi \eta)^{-1} \beta=\bar{\alpha} \tau_{G}$. One has $\mu \beta(x)=\lambda \psi \beta(x)=\lambda \alpha \tau_{G}=r^{\tau_{G}(x)} r^{-1}$, whence $(\beta, r) \in \operatorname{Der}\left(F_{G},(B, \mu)\right)$ and $(\bar{\alpha}, r) \in$ $\operatorname{Der}(G,(B, \mu))$. Evidently, $\psi^{1}([(\bar{\alpha}, r)])=[(\alpha, r)]$.

The rest of the proof repeats with minor modifications the proof of the exactness of the cohomology sequence for a coefficient short exact sequence of crossed $G$ modules [8].

Clearly for a short exact sequence of crossed $G$-modules we recover the known exact cohomology sequence $[\mathbf{6}, \mathbf{8}]$. Note also that Theorem 3.6 remains true for arbitrary $G$-partially crossed $P$-modules but in this case $\varphi^{1}, \psi^{1}$ and $\delta^{1}$ are maps of pointed sets.

Now for any partially crossed $G$-module $(A, \mu)$ the second cohomology $H^{2}(G, A)$ will be described in terms of extensions of groups.

Definition 3.7. An extension of $G$ by a partially crossed $G$-module $(A, \mu)$ is a pair $E=(1 \rightarrow A \stackrel{\sigma}{\rightarrow} X \stackrel{\psi}{\rightarrow} G \longrightarrow 1, \gamma)$, where $1 \longrightarrow A \stackrel{\sigma}{\rightarrow} X \stackrel{\psi}{\rightarrow} G \longrightarrow 1$ is a short exact sequence of groups, $\gamma$ is a section of $\psi$, that is $\psi \gamma=1_{G}$, one has the equality

$$
{ }^{g} a=\sigma^{-1}\left(\gamma(g) \sigma(a) \gamma(g)^{-1}\right)
$$

for $a \in A, g \in G$, and the following additional condition holds: 


\section{$\operatorname{Ker} \bar{\psi} \subset Z(A)$}

$\bar{\psi}$ being the restriction of $\psi$ on the subgroup of $X$ generated by $\gamma(G)$.

ExAmple 3.8. Let $A \rtimes G$ be the semidirect product of $A$ and $G$. Then one has an exact sequence of groups

$$
1 \longrightarrow A \stackrel{\sigma_{0}}{\longrightarrow} A \rtimes G \stackrel{\psi_{0}}{\longrightarrow} G \longrightarrow 1
$$

where $\sigma_{0}(a)=(a, 1), \psi_{0}(a, g)=g$. Take the canonical section $\gamma_{0}$ of $\psi_{0}$ given by $\gamma_{0}(g)=(1, g)$. It is easy to see that the pair $\left(1 \longrightarrow A \stackrel{\sigma_{0}}{\rightarrow} A \rtimes G \stackrel{\psi_{0}}{\rightarrow} G, \gamma_{0}\right)$ is an extension of $G$ by $(A, \mu)$, called trivial.

Definition 3.9. It will be said that $E=(1 \rightarrow A \stackrel{\sigma}{\rightarrow} X \stackrel{\psi}{\rightarrow} G \rightarrow 1, \gamma)$ is equivalent to $E^{\prime}=\left(1 \rightarrow A \stackrel{\sigma^{\prime}}{\rightarrow} X^{\prime} \stackrel{\psi^{\prime}}{\rightarrow} G \rightarrow 1, \gamma^{\prime}\right)$ if there exist a homomorphism $\vartheta: X \rightarrow X^{\prime}$ and an element $g \in G$ such that the diagram

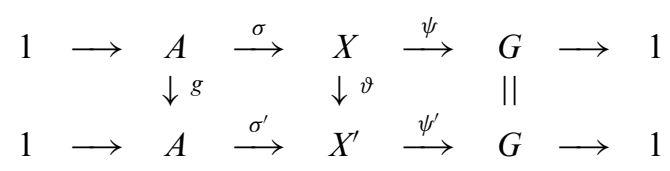

is commutative, $g: A \rightarrow A$ is the automorphism induced by the action of $g$ on $A$, and for any element $x \in G$ one has the equality

$$
\mu\left(\vartheta \gamma(x) \gamma^{\prime}(x)^{-1}\right)=g x g^{-1} x^{-1} .
$$

Clearly this relation $\sim$ is reflexive and symmetric. So for the relation $\sim$ to be an equivalence it remains to show the transitivity. Let $E \sim E^{\prime}$ and $E^{\prime} \sim E^{\prime \prime}$. Then the diagram

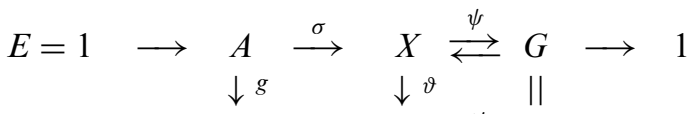

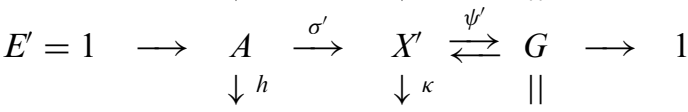

$$
\begin{aligned}
& E^{\prime \prime}=1 \longrightarrow A \stackrel{\sigma^{\prime \prime}}{\longrightarrow} X^{\prime \prime} \stackrel{\psi^{\prime \prime}}{\rightleftarrows} G \longrightarrow 1
\end{aligned}
$$

is commutative and one has the equalities

$$
\begin{aligned}
\mu\left(\vartheta \gamma(x) \gamma^{\prime}(x)^{-1}\right) & =g x g^{-1} x^{-1}, \\
\mu\left(\kappa \gamma^{\prime}(x) \gamma^{\prime \prime}(x)^{-1}\right) & =h x h^{-1} x^{-1} .
\end{aligned}
$$

We shall show that $\mu\left(\kappa \vartheta \gamma(x) \gamma^{\prime \prime}(x)^{-1}\right)=h g x g^{-1} h^{-1} x^{-1}$. Indeed, since

$$
\kappa \vartheta \gamma(x) \gamma^{\prime \prime}(x)^{-1}\left(\kappa \gamma^{\prime}(x) \gamma^{\prime \prime}(x)^{-1}\right)^{-1}=\kappa \vartheta \gamma(x) \kappa \gamma^{\prime}(x)^{-1}=\kappa\left(\vartheta \gamma(x) \cdot \gamma^{\prime}(x)^{-1},\right.
$$

one gets 


$$
\begin{aligned}
\mu\left(\kappa \vartheta(x) \gamma^{\prime \prime}(x)^{-1}\right) & =\mu \kappa\left(\vartheta \gamma(x) \gamma^{\prime}(x)^{-1} \mu \kappa \gamma^{\prime}(x) \gamma^{\prime \prime}(x)^{-1}\right) \\
& =h\left(g x g^{-1} x^{-1}\right) h^{-1} h x h^{-1} x^{-1} \\
& =h g x g^{-1} h^{-1} x^{-1} .
\end{aligned}
$$

Therefore the relation $\sim$ is an equivalence. Denote by $E^{1}(G, A)$ the set of equivalence classes of extensions of $G$ by the partially crossed $G$-module $(A, \mu)$.

THEOREM 3.10. There is a natural bijection

$$
\eta: H^{2}(G, A) \stackrel{\approx}{\rightarrow} E^{1}(G, A) .
$$

Proof. The map $\eta$ is defined as follows. For $[(\alpha, 1)] \in H^{2}(G, A)$ consider the diagram

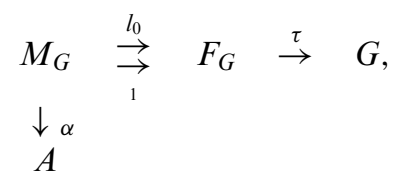

take the semidirect product $A \rtimes F_{G}, F_{G}$ acting on $A$ via $G$ and introduce an equivalence relation:

$$
(a, x) \sim\left(a^{\prime}, x^{\prime}\right) \stackrel{\rho}{\Longleftrightarrow} \tau(x)=\tau\left(x^{\prime}\right) \text { and } a=a^{\prime} \cdot \alpha\left(x, x^{\prime}\right) .
$$

In fact the equivalence $\rho$ is a congruence, since if $(a, x) \sim\left(a^{\prime}, x^{\prime}\right)$ and $(b, y) \sim\left(b^{\prime}, y^{\prime}\right)$, one has

$$
a^{x} b=a^{\prime} \alpha\left(x, x^{\prime}\right){ }^{x} b^{\prime}{ }^{x} \alpha\left(y, y^{\prime}\right)=a^{\prime x} b^{\prime} \alpha\left(x y, x^{\prime} y^{\prime}\right) .
$$

Denote $C=A \rtimes F_{G} / \rho$. One gets an exact sequence of groups

$$
1 \longrightarrow A \stackrel{\sigma}{\rightarrow} C \stackrel{\psi}{\rightarrow} G \longrightarrow 1
$$

where $\sigma(a)=[(a, 1)], \psi([(a, x)])=\tau(x)$ and the following diagram

$$
M_{G} \stackrel{\stackrel{l_{0}}{\rightarrow}}{\rightarrow} F_{G} \stackrel{\tau}{\rightarrow} G
$$

is commutative, $\delta(x)=[(1, x)], \sigma \alpha=\delta l_{1} \delta l_{0}^{-1}$.

Take a section $\gamma: G \rightarrow C$ given by $\gamma(g)=[(1,|g|)], g \in G$. It is easy to see that $\overline{\gamma(G)}=\operatorname{Im} \delta$. Therefore $\operatorname{Ker}\left(\left.\psi\right|_{\overline{\gamma(G)}}\right)=\delta(\operatorname{Ker} \tau)$. The equality $\sigma \alpha(1, x)=\delta(x)$, $x \in \operatorname{Ker} \tau$, implies $\operatorname{Ker}\left(\left.\psi\right|_{\overline{\gamma(G)}}\right) \subset Z(A)$ and it follows that the pair

$$
E=(1 \longrightarrow A \stackrel{\sigma}{\rightarrow} C \stackrel{\psi}{\rightarrow} G \longrightarrow 1, \gamma)
$$

is an extension of $G$ by $(A, \mu)$. 
Define $\eta([(\alpha, 1)])=[E]$. We have to show the well-definedness of $\eta$. Let $(\alpha, 1) \sim\left(\alpha^{\prime}, 1\right)$, meaning

$$
\alpha^{\prime}(x)=\beta l_{1}(x)^{-1 h} \alpha(x) \beta l_{0}(x), \quad x \in M_{G},
$$

for some $(\beta, h) \in \operatorname{Der}\left(F_{G},(A, \mu)\right)$ (see diagram 3.3$)$ and let $E^{\prime}=\left(1 \longrightarrow A \stackrel{\sigma^{\prime}}{\rightarrow} C^{\prime} \stackrel{\psi^{\prime}}{\rightarrow}\right.$ $\left.G \longrightarrow 1, \gamma^{\prime}\right)$ be the extension of $G$ by $(A, \mu)$ corresponding to $\left(\alpha^{\prime}, 1\right)$.

Define a map $v: A \rtimes F_{G} \longrightarrow A \rtimes G$ given by $v(a, x)=\left({ }^{h} a \beta(x), x\right)$. Thus $v$ provides a homomorphism, since $v((a, x)(b, y))=v\left(a^{x} b, x y\right)=\left({ }^{h}\left(a^{x} b\right) \beta(x y), x y\right)$ and $\nu(a, x) \nu(b, y)=\left({ }^{h} a \beta(x), x\right)\left({ }^{h} b \beta(y), y\right)=\left({ }^{h} a \beta(x){ }^{x}\left({ }^{h} b \beta(y)\right), x y\right)=\left({ }^{h} a^{h x} b \beta(x){ }^{x} \beta(y), x y\right)=$ $\left({ }^{h}\left(a^{x} b\right) \beta(x y), x y\right)$.

The homomorphism $v$ induces a homomorphism $v^{\prime}: A \rtimes F_{G} / \rho \rightarrow A \rtimes F_{G} / \rho^{\prime}$ given by $v^{\prime}\left([(a, x)]=[v(a, x)]\right.$. Indeed, let $(a, x) \stackrel{\rho}{\sim}\left(a^{\prime}, x^{\prime}\right)$, meaning $a=a^{\prime} \alpha\left(x, x^{\prime}\right)$ and $\tau(x)=\tau\left(x^{\prime}\right)$. We have to show that ${ }^{h} a \beta(x) \stackrel{\rho}{\sim}{ }^{h} a^{\prime} \beta\left(x^{\prime}\right)$, where $\alpha^{\prime}\left(x, x^{\prime}\right)=$ $\beta\left(x^{\prime}\right)^{-1 h} \alpha\left(x, x^{\prime}\right) \beta(x)$. One has

$$
{ }^{h} a \beta(x)={ }^{h} a^{\prime}{ }^{h} \alpha\left(x, x^{\prime}\right) \beta(x)={ }^{h} a^{\prime} \beta\left(x^{\prime}\right) \alpha^{\prime}\left(x, x^{\prime}\right) .
$$

Thus $v^{\prime}$ is a well-defined homomorphism and the diagram

$$
\begin{array}{rlll}
A & \triangleleft F_{G} & \longrightarrow & A \triangleleft F_{G} / \rho=C \\
& \downarrow v & & \downarrow v^{\prime} \\
A \triangleleft F_{G} & \longrightarrow & A \triangleleft F_{G} / \rho^{\prime}=C^{\prime}
\end{array}
$$

is commutative.

Now consider the following diagram

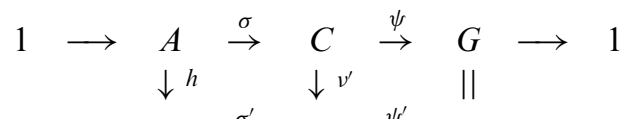

$$
\begin{aligned}
& 1 \longrightarrow A \stackrel{\sigma^{\prime}}{\rightarrow} \quad C^{\prime} \stackrel{\psi^{\prime}}{\rightarrow} \quad G \quad \longrightarrow \quad 1
\end{aligned}
$$

with sections $\gamma: G \rightarrow C, \gamma^{\prime}: G \rightarrow C^{\prime}$ defined as above. Clearly $\sigma^{\prime} h=v^{\prime} \sigma$ and $\left.v^{\prime} \gamma(x) \gamma(x)^{-1}=v^{\prime}((1,|x|)) \delta^{\prime}(x)=[(\beta(|x|),|x|)][(1,|x|)]^{-1}=[(\beta|x|), 1)\right]$. But $\mu \beta(|x|)=$ $h x h^{-1} x^{-1}$. It follows that $[E]=\left[E^{\prime}\right]$.

Conversely, define a map $\eta^{\prime}: E^{1}(G, A) \rightarrow H^{2}(G, A)$ as follows. Let $[E] \in E^{1}(G, A)$ and $E=(1 \rightarrow A \stackrel{\sigma}{\rightarrow} C \stackrel{\psi}{\rightarrow} G \rightarrow 1, \gamma)$. Then one gets a commutative diagram

$$
\begin{array}{ccccc}
M_{G} & \stackrel{l_{0}}{\rightarrow} & F_{G} & \stackrel{\tau}{\rightarrow} & G \\
\downarrow \alpha & l_{1} & \downarrow \delta & & \| \\
A & \stackrel{\sigma}{\rightarrow} & C & \stackrel{\psi}{\rightarrow} & G
\end{array}
$$

with $\delta$ induced by $\gamma$ and $\sigma \alpha=\delta l_{1} \delta l_{0}^{-1}$. Clearly $\alpha$ is a crossed homomorphism such that $\alpha(\Delta)=1$ and $\operatorname{Im} \alpha \subset Z(A)$. Define $\eta^{\prime}([E])=[(\alpha, 1)]$.

We have to show the well-definedness again. If $\delta^{\prime}$ is another homomorphism such that $\beta \delta^{\prime}=\tau$, then $\delta(y) \delta^{\prime}(y)^{-1} \in Z(A), y \in F_{G}$. Thus $\delta \delta^{\prime-1}$ induces a crossed homomorphism $\beta: F_{G} \rightarrow Z(A)$ and it is obvious that $\left(\alpha^{\prime}, 1\right)=\left(\beta l_{0}, 1\right)(\alpha, 1)\left(\beta l_{1}, 1\right)^{-1}$ with $(\beta, 1) \in \operatorname{Der}\left(F_{G}, A\right)$. 
Assume now that $E$ is equivalent to $E^{\prime}=\left(1 \rightarrow A \stackrel{\sigma^{\prime}}{\rightarrow} C^{\prime} \stackrel{\psi^{\prime}}{\rightarrow} G \rightarrow 1, \gamma^{\prime}\right)$ by the pair ( $h: A \rightarrow A, \vartheta: C \rightarrow C^{\prime}$ ) implying the equality $\mu\left(\vartheta \gamma(x) \gamma^{\prime}(x)^{-1}\right)=h x h^{-1} x^{-1}, x \in G$. By using the equalities $\psi \delta=\psi^{\prime} \delta^{\prime}=\tau, \sigma \alpha=\delta l_{1} \delta l_{0}^{-1}, \sigma^{\prime} \alpha^{\prime}=\delta^{\prime} l_{1} \delta^{\prime} l_{0}^{-1}, \sigma^{\prime} h=\vartheta \sigma$, one gets $\vartheta \delta=\sigma^{\prime} \beta \delta^{\prime}, \vartheta \delta l_{0}=\sigma^{\prime} \beta l_{0} \delta^{\prime} l_{0}, \vartheta \delta l_{1}=\sigma^{\prime} \beta l_{1} \delta^{\prime} l_{1}$, where $\beta: F_{G} \longrightarrow A$ is a crossed homomorphism induced by $\vartheta \delta \delta^{\prime-1}$.

Clearly $(\beta, h)$ is an element of $\operatorname{Der}\left(F_{G}, A\right)$. Further, $\sigma \alpha \delta l_{0}=\delta l_{1}$, so one has $\vartheta \sigma \alpha \vartheta \delta l_{0}=\vartheta \delta l_{1}$ and the following equalities

$$
\begin{aligned}
\vartheta \sigma \alpha \sigma^{\prime} \beta l_{0} \delta^{\prime} l_{0} & =\sigma^{\prime} \beta l_{1} \delta^{\prime} l_{1}, \\
\vartheta \sigma \alpha \sigma^{\prime} \beta l_{0} & =\sigma^{\prime} \beta l_{1} \delta^{\prime} l_{1} \delta^{\prime} l_{0}^{-1}, \\
\sigma^{\prime} h \alpha \sigma^{\prime} \beta l_{0} & =\sigma^{\prime} \beta l_{1} \sigma^{\prime} \alpha^{\prime} .
\end{aligned}
$$

Finally $\alpha^{\prime}=\beta l_{1}^{-1} h \alpha \beta l_{0}$.

Therefore $\left(\alpha^{\prime}, 1\right)$ is equivalent to $(\alpha, 1)$. It is easily checked that $\eta \eta^{\prime}$ and $\eta^{\prime} \eta$ are identity maps.

4. Higher non-abelian cohomology. Let $(A, \mu)$ be a $G$-partially crossed $P$-module. Take the free cotriple resolution $F_{*}(G)$ of the group $G$ :

$$
\cdots \underset{\vdots}{\rightarrow} F_{n+1} \underset{\partial_{n+1}^{n+1}}{\stackrel{\partial_{0}^{n+1}}{\longrightarrow}} F_{n} \underset{\partial_{n}^{n}}{\stackrel{\partial_{0}^{n}}{\rightarrow}} \cdots \underset{\vdots}{\rightarrow} F_{2} \underset{\partial_{2}^{2}}{\stackrel{\partial_{0}^{2}}{\rightarrow}} F_{1} \stackrel{\tau_{1}}{\rightarrow} M_{0} \underset{\partial_{1}^{1}}{\stackrel{\partial_{0}^{1}}{\rightarrow}} F_{0} \longrightarrow G
$$

with $F_{n}=F^{n+1}(A), \quad n \geq 0, \quad F_{0}=F(A)$ the free group generated by $A$ and $F^{n+1}(A)=F\left(F^{n}(A)\right), \partial_{i}^{n}=F^{i} \tau F^{n-i}, s_{i}^{n}=F^{i} \delta F^{n-i}$, where $\delta: F(A) \longrightarrow F^{2}(A)$ is induced by the canonical inclusion $A \rightarrow F(A)$. Clearly $(A, \mu)$ can be viewed as an $F_{n}$-partially crossed $P$-module induced by $\tau \partial_{0}^{1} \partial_{0}^{2} \cdots \partial_{0}^{n}$. Therefore the group $\operatorname{Der}\left(F_{n},(A, \mu)\right)$, $n \geq 0$, is defined. Denote by $\widetilde{Z}^{1}\left(F_{n},(A, \mu)\right)$ the subset of $\operatorname{Der}\left(F_{n},(A, \mu)\right)$ consisting of all elements of the form $(\alpha, 1)$ for $n$ odd and of the form $(\alpha, r)$ for $n$ even satisfying the condition

$$
\prod_{j=0}^{n+1}\left(\alpha \partial_{j}^{n+1}\right)^{\varepsilon_{j}}=1, \quad \varepsilon_{j}=(-1)^{j}
$$

Since $\mu \alpha(x)=1$ for any $x \in F_{n}$ and for $n$ odd, in this case we have $\alpha\left(F_{n}\right) \subset Z(A)$, $n \geq 1$. In the set $\widetilde{Z}^{1}\left(F_{n},(A, \mu)\right), n \geq 1$, a relation $\sim$ is introduced as follows:

$\left(\alpha^{\prime}, 1\right) \sim(\alpha, 1)$ for $n$ odd and $\left(\alpha^{\prime}, r^{\prime}\right) \sim(\alpha, r)$ for $n$ even if there is an element $(\beta, h) \in \operatorname{Der}\left(F_{n-1},(A, \mu)\right)$ such that

$$
\alpha^{\prime}(x)={ }^{h} \alpha(x) \prod_{i=0}^{n}\left(\beta \partial_{i}^{n}(x)\right)^{\varepsilon_{i}}, \quad \varepsilon_{i}=(-1)^{i}, \quad x \in F_{n},
$$

and $r^{\prime}=r$ for $n$ even.

The homomorphism $\tau \partial_{i_{n}}^{1} \partial_{i_{n-1}}^{2} \cdots \partial_{i_{2}}^{n-1} \partial_{i_{1}}^{n}$ does not depend of the sequence $\left(i_{1}, i_{2}\right.$, $\left.\cdots, i_{n-1}, i_{n}\right)$ implying

$$
\beta \partial_{j}^{n}(x)\left(\beta \partial_{l}^{n}(x)\right)^{-1}=\left(\beta \partial_{l}^{n}(x)\right)^{-1}\left(\beta \partial_{j}^{n}(x) \in \operatorname{Ker} \mu, \quad x \in F_{n},\right.
$$


for $0 \leq j, l \leq n$. It follows that the product $\prod_{i=0}^{n}\left(\beta \partial_{i}^{n}(x)\right)^{\varepsilon_{i}}, \varepsilon_{i}=(-1)^{i}$, does not depend on the order of the factors. Obviously the aforedefined relation is an equivalence.

Definition 4.1. The higher non-abelian cohomology of a group $G$ with coeffcients in a $G$-partially crossed $P$-module $(A, \mu)$ is given by

$$
H^{n+1}(G,(A, \mu))=\widetilde{Z^{1}}\left(F_{n},(A, \mu)\right) / \sim, \quad n \geq 1 .
$$

It is easily checked that for $n=1$ we recover the second cohomology set of $G$ with coefficients in $(A, \mu)$.

The map $H^{n+1}(G, \operatorname{Ker} \mu) \rightarrow H^{n+1}(G,(A, \mu))$ given by $[f] \mapsto[(f, 1)]$ is surjective and is bijective if $\mu: A \rightarrow P$ is the trivial homomorphism (in this case $A$ is abelian).

In order to express this in terms of the derived functors of the group of derivations with respect to the contravariant variable, cohomotopy pointed sets of some cosimplicial groups will be introduced.

Let

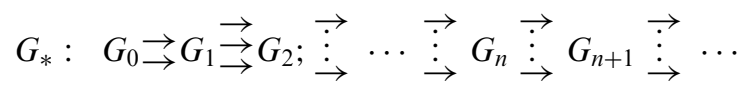

be a cosimplicial group. Clearly $\partial_{j}^{n} \partial_{i}^{n-1}=\partial_{i}^{n} \partial_{j-1}^{n-1}, i<j$.

Assume that $G_{*}$ satisfies the following condition:

(a) Denote by $L_{n+1}$ the subgroup of $G_{n+1}$ generated by $\bigcup_{i=0}^{n+1} \partial_{i}^{n}\left(G_{n}\right)$. Then for any element $x \in G_{n}, n \geq 0$, the product $\partial_{i}^{n}(x) \partial_{j}^{n}(x)^{-1}, 0 \leq i, j \leq n+1$, commutes with every element of $L_{n+1}$.

In particular it follows that one has the equality $\partial_{i}^{n}(x) \partial_{j}^{n}(x)^{-1}=\partial_{j}^{n}(x)^{-1} \partial_{i}^{n}(x)$, $0 \leq i, j \leq n, n \geq 0$.

Under this condition the cosimplicial group $G_{*}$ induces a group chain complex

$$
1 \longrightarrow G_{0} \stackrel{d_{0}}{\rightarrow} G_{1} \stackrel{d_{1}}{\rightarrow} G_{2} \stackrel{d_{2}}{\rightarrow} \cdots G_{n} \stackrel{d_{n}}{\rightarrow} G_{n+1} \stackrel{d_{n+1}}{\longrightarrow} \cdots
$$

with $d_{n}(x)=\prod_{i=0}^{n+1} \partial_{i}^{n}(x)^{\varepsilon_{i}}, \varepsilon_{i}=(-1)^{i}, n \geq 0$. It is easily checked that the maps $d_{n}$, $n \geq 0$, are homomorphisms and $d_{n} d_{n-1}=0, n \geq 1$.

Definition 4.2. The right quotient sets $\operatorname{Ker} d_{n} / \operatorname{Im} d_{n-1}$ will be called cohomotopy sets $\pi_{n}\left(G_{*}\right), n \geq 0$, of the cosimplicial group $G_{*}$.

It is obvious that for abelian cosimplicial groups we recover the well known homology groups.

\section{Proposition 4.3. Let}

$$
1 \longrightarrow G_{*}^{\prime} \longrightarrow G_{*} \longrightarrow G_{*}^{\prime \prime} \longrightarrow 1
$$

be a short exact sequence of cosimplicial groups satisfying condition (a). Then there is a long exact sequence of pointed cohomotopy sets

$$
\begin{aligned}
1 \longrightarrow \pi_{0}\left(G_{*}^{\prime}\right) \longrightarrow \pi_{0}\left(G_{*}\right) \longrightarrow \pi_{0}\left(G_{*}^{\prime \prime}\right) \longrightarrow \pi_{1}\left(G_{*}^{\prime}\right) \longrightarrow \cdots \longrightarrow \pi_{n-1}\left(G_{*}^{\prime \prime}\right) \\
\longrightarrow \pi_{n}\left(G_{*}^{\prime}\right) \longrightarrow \pi_{n}\left(G_{*}\right) \longrightarrow \pi_{n}\left(G_{*}^{\prime \prime}\right) \longrightarrow \pi_{n+1}\left(G_{*}^{\prime}\right) \longrightarrow \cdots
\end{aligned}
$$


Proof. Straightforward.

This definition of cohomotopy pointed sets defined for cosimplicial groups satisfying the aforementioned condition allows us to define cotriple right derived functors of some group valued contravariant functors.

Let $\mathbf{A}$ be an arbitrary category and $\mathbf{F}=(\mathbf{F}, \tau, \delta)$ be a cotriple in the category $\mathbf{A}$. For any object $A \in \mathrm{ob} \mathbf{A}$ take its cotriple resolution:

$$
F_{*}(A) \stackrel{\tau}{\rightarrow} A .
$$

similarly to the case of groups considered above.

Let $T: \mathbf{A} \rightarrow \mathbf{G r}$ be a contravariant functor to the category of groups satisfying the following condition:

(b) the product $T \partial_{i}^{n+1}(x) T \partial_{j}^{n+1}(x)^{-1}, 0 \leq i, j \leq n+1, n \geq 0$, commutes with every element of the subgroup of $T F_{n+1}(A)$ generated by $\bigcup_{i=0}^{n+1} \operatorname{Im} T \partial_{i}^{n+1}, n \geq 0$.

Definition 4.4. The right derived functors of the contravariant functor $T$ with respect to the cotriple $\mathbf{F}$ are the pointed sets

$$
R_{\mathrm{F}}^{n} T(A)=\pi_{n}\left(T F_{*}(A)\right), \quad n \geq 0, \quad A \in \mathrm{ob} \mathbf{A} .
$$

If $f: A \rightarrow A^{\prime}$ is a morphism of the category $\mathbf{A}$, then one gets a morphism $T F_{*}(f): T F_{*}\left(A^{\prime}\right) \rightarrow T F_{*}(A)$ inducing maps of pointed sets $R_{\mathbf{F}}^{n} T(f)=\pi_{n} T F_{*}(f)$ : $R_{\mathbf{F}}^{n} T\left(A^{\prime}\right) \rightarrow R_{\mathbf{F}}^{n} T(A), n \geq 0$.

REMARK 4.5. One can define similarly the right derived functors $R_{\mathbf{L}}^{n} T$ with respect to a triple $\mathbf{L}$ in the category $\mathbf{A}$ for group valued covariant functors $T$ satisfying the same condition.

As noted above the main application of these derived functors will be their close relationship with non-abelian cohomology of groups.

Let $(A, \mu)$ be a $G$-partially crossed $P$-module and consider the free cotriple resolution $F_{*}(G)$ of the group $G$ (see (4.1)). In general the cosimplicial group $\operatorname{Der}\left(F_{*}(G),(A, \mu)\right)$ does not verify condition (a), in other words the contravariant functor $\operatorname{Der}(-,(A, \mu))$ from the category of groups acting on $(A, \mu)$ to the category of groups $\mathbf{G r}$ does not verify condition (b). Below it will be shown that for a wide class of coefficients $(A, \mu)$ condition (b) holds for the functor $\operatorname{Der}(-,(A, \mu))$.

One gets a sequence of groups and maps

$$
\begin{aligned}
1 \longrightarrow \operatorname{Der}\left(F_{0},(A, \mu)\right) \stackrel{d_{0}}{\rightarrow} \operatorname{Der}\left(F_{1},(A, \mu)\right) \stackrel{d_{1}}{\rightarrow} \cdots \longrightarrow \\
\longrightarrow \operatorname{Der}\left(F_{n},(A, \mu) \stackrel{d_{n}}{\rightarrow} \operatorname{Der}\left(F_{n+1},(A, \mu)\right) \stackrel{d_{n+1}}{\rightarrow} \cdots\right.
\end{aligned}
$$

with $d_{n}((\alpha, r))(x)=(\gamma, s)$, where $\gamma=\prod_{i=0}^{n+1}\left(\alpha \partial_{i}^{n+1}\right)^{\varepsilon_{i}}, \quad \varepsilon_{i}=(-1)^{i}, \quad(\alpha, r) \in \operatorname{Der}\left(F_{n}\right.$, $(A, \mu)), x \in F_{n+1}, s=1$ for $n$ even and $s=r$ for $n$ odd.

Introduce in $\operatorname{Ker} d_{n}, n \geq 0$, an equivalence by $\left(\alpha^{\prime}, r^{\prime}\right) \sim(\alpha, r) \Longleftrightarrow$ there exists $(\beta, h) \in \operatorname{Der}\left(F_{n-1},(A, \mu)\right)$ such that $\alpha^{\prime}(x)={ }^{h} \alpha(x) \prod_{i=0}^{n} \beta \partial_{i}^{n}(x)^{\varepsilon_{i}}, \varepsilon_{i}=(-1)^{i}, r^{\prime}=r$. Clearly the quotient sets coincide with $H^{n+1}(G,(A, \mu)), n \geq 1$. It is easily checked that one has $\operatorname{Ker} d_{0} \approx \operatorname{Der}(G,(A, \mu))$. Moreover, the composite $d_{n} d_{n-1}, n \geq 1$, is the trivial map and the maps $d_{n}$ are homomorphisms for $n$ odd. 
Proposition 4.6. Let $(A, \mu)$ be a G-partially crossed P-module such that $P$ acts trivially on $\operatorname{Ker} \mu$. Then the maps $d_{n}$ of the sequence (4.2) are homomorphisms for all $n \geq 0$. Moreover, in this case $\operatorname{Im} d_{n}$ belongs to the center of $\operatorname{Der}\left(F_{n+1},(A, \mu)\right)$ for $n$ even and $\operatorname{Ker} d_{n}$ belongs to the center of $\operatorname{Der}\left(F_{n},(A, \mu)\right)$ for $n$ odd.

Proof. Use the equalities $\alpha \partial_{i}^{n+1}(x) \alpha \partial_{j}^{n+1}(x)^{-1}=\alpha \partial_{j}^{n+1}(x)^{-1} \alpha \partial_{i}^{n+1}(x), 0 \leq i, j \leq$ $n+1$, for $(\alpha, r) \in \operatorname{Der}\left(F_{n},(A, \mu)\right)$ and the fact that in this case any element of the form $(\alpha, 1) \in \operatorname{Der}\left(F_{n},(A, \mu)\right)$ belongs to the center.

Theorem 4.7. Let $(A, \mu)$ be a G-partially crossed P-module with $P$ acting trivially on $\operatorname{Ker} \mu$ and let $\mathbf{F}$ be the free cotriple in the category of groups acting on $(A, \mu)$.

(i) One has isomorphisms

$$
\begin{aligned}
& R_{\mathbf{F}}^{0} \operatorname{Der}(G,(A, \mu)) \approx \operatorname{Der}(G,(A, \mu)), \\
& R_{\mathbf{F}}^{n} \operatorname{Der}(G,(A, \mu)) \approx H^{n+1}(G,(A, \mu)), \quad n \geq 1,
\end{aligned}
$$

and $H^{n+1}(G,(A, \mu))$ is an abelian group for $n$ odd.

(ii) Any short exact sequence of G-partially crossed P-modules

$$
1 \longrightarrow\left(A^{\prime}, \mu^{\prime}\right) \stackrel{\varphi}{\rightarrow}(A, \mu) \stackrel{\psi}{\rightarrow}\left(A^{\prime \prime}, \mu^{\prime \prime}\right) \longrightarrow 1
$$

with $P$ acting trivially on $\operatorname{Ker} \mu^{\prime}$, Ker $\mu$ and Ker $\mu^{\prime \prime}$, induces a long exact cohomology sequence

$$
\begin{aligned}
1 \longrightarrow \operatorname{Der}\left(G,\left(A^{\prime}, \mu^{\prime}\right)\right) \longrightarrow \operatorname{Der}(G,(A, \mu)) \longrightarrow \operatorname{Der}\left(G,\left(A^{\prime \prime}, \mu^{\prime \prime}\right)\right) \longrightarrow \\
\longrightarrow H^{2}\left(G,\left(A^{\prime}, \mu^{\prime}\right)\right) \longrightarrow H^{2}(G,(A, \mu)) \longrightarrow H^{2}\left(G,\left(A^{\prime \prime}, \mu^{\prime \prime}\right)\right) \longrightarrow \\
\longrightarrow H^{3}\left(G,\left(A^{\prime}, \mu^{\prime}\right)\right) \longrightarrow \cdots \longrightarrow H^{n-1}\left(G,\left(A^{\prime \prime}, \mu^{\prime \prime}\right)\right) \longrightarrow \\
\longrightarrow H^{n}\left(G,\left(A^{\prime}, \mu^{\prime}\right)\right) \longrightarrow H^{n}(G,(A, \mu)) \longrightarrow H^{n}\left(G,\left(A^{\prime \prime}, \mu^{\prime \prime}\right)\right) \longrightarrow \\
\longrightarrow H^{n+1}\left(G,\left(A^{\prime}, \mu^{\prime}\right)\right) \longrightarrow \cdots .
\end{aligned}
$$

Proof. Since the functor $\operatorname{Der}(-,(A, \mu))$ satisfies condition (b) with respect to the free cotriple $\mathbf{F}$, the right derived functors of $\operatorname{Der}(-,(A, \mu))$ are well defined, the sequence (4.2) becomes a complex of non-abelian groups and the statement of (i) follows from Proposition 4.6.

The short exact coefficient sequence of (ii) induces a short exact sequence of cosimplicial groups

$$
1 \longrightarrow \operatorname{Der}\left(F_{*}(G),\left(A^{\prime}, \mu^{\prime}\right)\right) \longrightarrow \operatorname{Der}\left(F_{*}(G),(A, \mu)\right) \longrightarrow \operatorname{Der}\left(F_{*}(G),\left(A^{\prime \prime}, \mu^{\prime \prime}\right)\right) \rightarrow 1
$$

satisfying condition (a). It remains to apply Proposition 4.3 to get the required long exact cohomology sequence.

The definition of non-abelian cohomology with coefficients in $G$-partially crossed $P$-modules allows as to introduce the definition of higher non-abelian cohomology of a group $G$ with coefficients in any $G$-group. This can be done as follows.

Let $A$ be an arbitrary $G$-group, meaning the group $G$ acts on the left on the group $A$. Take the quotient group $P=A / Z(A)$. Define an action of $P$ on $A$ and an action of $G$ on $P$ as follows: 


$$
\begin{aligned}
& { }^{\left[a^{\prime}\right]} a={ }^{a^{\prime}} a, \quad\left[a^{\prime}\right] \in P, \quad a, a^{\prime} \in A, \\
& { }^{g}[a]=\left[{ }^{g} a\right], \quad g \in G, \quad a \in A .
\end{aligned}
$$

Let $\mu_{A}: A \longrightarrow P$ be the canonical homomorphism. It is easily checked that the pair $\left(A, \mu_{A}\right)$ is a $G$-crossed $P$-module under the aforedefined actions.

Definition 4.8. The $n$-th cohomology $H^{n}(G, A), n \geq 0$, of the group $G$ with coefficients in a $G$-group $A$ is given by

$$
H^{n}(G, A)=H^{n}\left(G,\left(A, \mu_{A}\right)\right), \quad n \geq 0 .
$$

For $n=1$ this cohomology differs from the first pointed set cohomology defined in $[\mathbf{1 0}]$.

Let

$$
1 \longrightarrow A \stackrel{\varphi}{\rightarrow} B \stackrel{\psi}{\rightarrow} C \longrightarrow 1
$$

be a central extension of $G$-groups. Then $\psi$ induces an isomorphism of $G$-groups $\vartheta: B / Z(B) \stackrel{\approx}{\rightarrow} C / \psi(Z(B))$ and one gets a short exact sequence of $G$-crossed $P$-modules with $P=B / Z(B)$

$$
1 \longrightarrow(A, 1) \stackrel{\varphi}{\rightarrow}\left(B, \mu_{B}\right) \stackrel{\psi}{\rightarrow}\left(C, \bar{\mu}_{C}\right) \longrightarrow 1,
$$

where $\bar{\mu}_{C}$ is the composite of the canonical map $C \rightarrow C / \psi(Z(B))$ and the isomorphism $\vartheta^{-1}$.

Corollary 4.9. Any central extension of G-groups

$$
1 \longrightarrow A \stackrel{\varphi}{\rightarrow} B \stackrel{\psi}{\rightarrow} C \longrightarrow 1
$$

induces a long exact cohomology sequence

$$
\begin{aligned}
& 1 \rightarrow H^{0}(G, A) \stackrel{\varphi^{0}}{\rightarrow} H^{0}(G, B) \stackrel{\psi^{0}}{\rightarrow} H^{0}(G, C) \stackrel{\delta^{0}}{\rightarrow} H^{1}(G, A) \stackrel{\varphi^{1}}{\rightarrow} H^{1}(G, B) \\
& \stackrel{\psi^{1}}{\rightarrow} H^{1}\left(G,\left(C, \bar{\mu}_{C}\right)\right) \stackrel{\delta^{1}}{\rightarrow} H^{2}(G, A) \stackrel{\varphi^{2}}{\rightarrow} H^{2}(G, B) \stackrel{\psi^{2}}{\rightarrow} H^{2}\left(G,\left(C, \bar{\mu}_{C}\right)\right) \\
& \stackrel{\delta^{2}}{\rightarrow} H^{3}(G, A) \stackrel{\varphi^{3}}{\rightarrow} \cdots \longrightarrow H^{n-1}\left(G,\left(C, \bar{\mu}_{C}\right)\right) \stackrel{\delta^{n-1}}{\rightarrow} H^{n}(G, A) \\
& \stackrel{\varphi^{n}}{\rightarrow} H^{n}(G, B) \stackrel{\psi^{n}}{\rightarrow} H^{n}\left(G,\left(C, \bar{\mu}_{C}\right)\right) \stackrel{\delta^{n}}{\rightarrow} H^{n}(G, A) \longrightarrow \cdots
\end{aligned}
$$

Proof. Clearly in the induced short exact sequence of $G$-crossed $P$-modules the group $P$ acts trivially on $\operatorname{Ker} \mu_{B}$ and $\operatorname{Ker} \bar{\mu}_{C}$. So we can apply Theorem 4.7 giving together with Theorem 3.6 the required long exact cohomology sequence.

ACKNOWLedgements. The work was partially supported by INTAS Georgia grant No 213 and Nato Science Program No 975316. 


\section{REFERENCES}

1. R. Brown, D. Johnson and E. F. Robertson, Some computations of the non-abelian tensor products of groups, J. Algebra 111 (1987), 177-202.

2. M. Bullejos and A. Cegarra, A 3-dimentional cohomology of groups with applications to homotopy classification of continuous maps, Canad. J. Math. 43 (1991), 265-296.

3. A. M. Cegarra and A. R. Garzón, A long exact sequence in non-abelian cohomology, in Category Theory (Como, 1990), Lecture Notes in Math. No 1488 (Springer-Verlag, 1991), 79-94.

4. R. Dedecker, Cohomologie non abelienne, Seminaire de l'Institut Mathematique Lille, 1963-1964.

5. D. Guin, Cohomologie et homologie non abeliennes des groupes, C. R. Acad. Sci. Paris 301 (1985), Serie 1, No 7.

6. D. Guin, Cohomologie et homologie non abeliennes des groupes, J. Pure Appl. Algebra 50 (1988), 109-137.

7. H. Inassaridze, Non-abelian homological algebra and its applications (Kluwer Academic Publishers, 1997).

8. H. Inassaridze, Non-abelian cohomology of groups, Georgian Math. J. 4, No 4 (1997), 313-332.

9. J.-L. Loday, Cyclic homology (Springer-Verlag, 1997).

10. J.-P. Serre, Cohomologie galoisienne, Lecture Notes in Mathematics No. 5. (Springer-Verlag, 1964). 